INLO-PUB-22/99, YITP-SB-99-70

\title{
Bottom quark electroproduction in variable flavor number schemes
}

\author{
A. Chuvakin, J. Smith \\ C.N. Yang Institute for Theoretical Physics, \\ State University of New York at Stony Brook, \\ New York 11794-3840, USA. \\ W.L. van Neerven \\ Instituut-Lorentz, \\ University of Leiden, PO Box 9506, 2300 RA Leiden, The Netherlands.
}

(November 6, 2018)

\begin{abstract}
Two variable flavor number schemes are used to describe bottom quark production in deep inelastic electron-proton scattering. In these schemes the coefficient functions are derived from mass factorization of the heavy quark coefficient functions presented in a fixed flavor number scheme. Also one has to construct a parton density set with five light flavors $(\mathrm{u}, \mathrm{d}, \mathrm{s}, \mathrm{c}, \mathrm{b})$ out of a set which only contains four light flavors $(\mathrm{u}, \mathrm{d}, \mathrm{s}, \mathrm{c})$. In order $\alpha_{s}^{2}$ the two sets are discontinuous at $\mu=m_{b}$ which follows from mass factorization of the heavy quark coefficient functions when it is carried out in the $\overline{\mathrm{MS}}$-scheme. Both variable flavor number schemes give almost identical predictions for the bottom structure functions $F_{2, b}$ and $F_{L, b}$. Also they both agree well with the corresponding results based on fixed order four-flavor perturbation theory over a wide range in $x$ and $Q^{2}$.
\end{abstract}

PACS 11.10Jj, 12.38Bx, 13.60Hb, 13.87Ce.

Typeset using REVTEX 


\section{INTRODUCTION}

The H1 and ZEUS experiments at HERA now have enough integrated luminosity to study bottom quark deep inelastic electroproduction. Therefore it is an appropriate time to present up-to-date predictions for the bottom quark components of the deep inelastic structure functions $F_{i}\left(x, Q^{2}\right)$ where $i=2, L$. We assume that the bottom quark is produced in an extrinsic fashion and that the neutral current reaction dominates over the charged current one. This means that in fixed order perturbative QCD the heavy quark structure functions $F_{i, b}\left(x, Q^{2}, m_{b}^{2}\right), i=2, L$ are given by the virtual-photon gluon-fusion processes and their higher order corrections with only light partons in the initial state. Notice that in the case of bottom quark production the light partons are represented by the gluon and the four light flavors $\mathrm{u}, \mathrm{d}, \mathrm{s}, \mathrm{c}$ together with their anti-particles.

In the literature one has adopted two different treatments of extrinsic bottom quark production, which are known as the massive and massless descriptions. The former treats the bottom quark as a heavy quark (with mass $m_{b}$ ) and the partonic cross sections (or heavy quark coefficient functions) are described by fixed order perturbation theory (FOPT) as mentioned above. Notice that due to the work in [1] the perturbation series is now known up to second order. The latter treatment, which has been rather popular among groups which fit parton densities to experimental data, treats the bottom quark as a massless quark so that it can be represented by a scale dependent parton density $f_{b}\left(x, \mu^{2}\right)$. Although at first sight these approaches are completely different they are actually intimately related. It was shown in [2] that the large logarithms of the type $\ln \left(Q^{2} / m_{b}^{2}\right)$, which appear in FOPT when $Q^{2} \gg m_{b}^{2}$, can be resummed in all orders. The upshot of this procedure is that the bottom components of the deep inelastic structure functions $F_{i, b}\left(x, Q^{2}, m_{b}^{2}\right)$, where $i=$ $2, L$, which in the FOPT approach are written as convolutions of heavy quark coefficient functions with four-flavor light-mass $(\mathrm{u}, \mathrm{d}, \mathrm{s}, \mathrm{c})$ parton densities, become, after resummation, convolutions of light-mass parton coefficient functions with five-flavor light-mass parton densities which also include a bottom quark density. This procedure leads to the so-called zero mass variable flavor number scheme (ZM-VFNS) for $F_{i, b}\left(x, Q^{2}\right)$ where the mass of the bottom quark is absorbed into the new five flavor densities. To implement this scheme one has to be careful to use quantities which are collinearly finite in the limit $m_{b} \rightarrow 0$. From the above considerations it is clear that the FOPT approach is better when the bottom quark pair is produced near threshold (where $Q^{2}\left(x^{-1}-1\right) \geq 4 m_{b}^{2}$ ) because terms in $m_{b}$ are important in this kinematic region. On the other hand far above threshold, where also $Q^{2} \gg m_{b}^{2}$, the large logarithms mentioned above dominate the structure functions so that the ZM-VFNS approach should be more appropriate. Both approaches are characterized by the number of active flavors involved in the description of the parton densities which are given by four and five respectively. Each scheme has different densities but the momentum sum rule either gets contributions from four-flavor densities or five-flavor densities and is always satisfied.

As most of the experimental data will be in the kinematical regime which is between the threshold and the large $Q^{2}$ region, a third approach, called the variable flavour number scheme (VFNS), has been introduced to describe the heavy quark components $F_{i, b}\left(x, Q^{2}\right)$ of the deep inelastic structure functions. Actually there are several such schemes. They include

the Aivasis, Collins, Olness, Tung (ACOT) [3], scheme, the Buza, Matiounine, Smith, van 
Neerven (BMSN) [2], [4] scheme, the Thorne, Roberts (TR) [5] scheme and the Chuvakin, Smith, van Neerven (CSN) [6] scheme. A discussion of them is given in the last reference. The difference between the schemes can be attributed to two ingredients entering in their construction. The first one is the mass factorization procedure carried out before the large logarithms can be resummed. The second one is the matching condition imposed on the heavy quark density, which has to vanish in the threshold region of the production process. Another aspect of these approaches is that one needs two sets of parton densities. For bottom quark production one set only contains densities in a four-flavor number scheme whereas the second one, which also includes a bottom quark density, is parametrized in a five-flavor number scheme. Both parameterizations have to satisfy the matching relations quoted in [2]. Up to next-to-leading order (NLO) they are continuous at the scale $\mu=m_{b}$ whereas in next-to-next-to leading order (NNLO) the parton densities become discontinuous while going from a four to a five flavor scheme. Starting from a three-flavor set of parton densities given in [7] we have recently constructed in [8] a four-flavor set of densities which satisfied the matching relations in [2] at the scale $\mu=m_{c}$. Then we evolved these densities with LO or NLO splitting functions up to the scale $\mu=m_{b}$ and constructed a five-flavor set which also satisfied the matching relations in [2]. This set was further evolved with LO and NLO splitting functions up to high scales. Notice that since the NNLO splitting functions are unknown the only difference between the NLO and NNLO parton densities can be attributed to the boundary conditions at $\mu=m_{c}$ and $\mu=m_{b}$ where the latter densities become discontinuous contrary to the LO and NLO ones. We can now use these densities to discuss VFNS for bottom quark deep inelastic electroproduction, in particular in the CSN and BMSN schemes. The previous discussions in [6] were focussed on applications to charm quark electroproduction.

Since any description for bottom quarks follows closely that for charm quarks we refer the interested reader to [6] for most of the details and simply specialize to bottom quark electroproduction in Sec.II. We work to second order in the running coupling constant $\alpha_{s}\left(\mu^{2}\right)$. Numerical results are shown for the structure functions $F_{2, b}$ and $F_{L, b}$ in the CSN and BMSN schemes. 


\section{BOTTOM QUARK STRUCTURE FUNCTIONS}

In this Section we consider bottom quark deep inelastic electroproduction in two variable flavor number schemes, namely the BMSN scheme as proposed in [2], [4] and in the CSN scheme as proposed in [6]. For that purpose we have constructed in [8] a five-flavor parton density set from a four-flavor parton density set. Using our densities we will study the differences between the bottom components of the deep inelastic structure functions $F_{i, b}^{\mathrm{CSN}}\left(n_{f}+1\right)$ and $F_{i, b}^{\mathrm{BMSN}}\left(n_{f}+1\right)$, where the number of light flavors is $n_{f}=4$.

To keep the discussion short we simply refer the reader to Sec.III of [6] for a discussion of the $\overline{\mathrm{MS}}$ parton densities, the exact solution for the running coupling constant and the scale choice. All references to three-flavour (four-flavor) densities should be replaced by four-flavor (five-flavor) densities respectively. All our calculations of next-to-leading (NLO) and nextto-next-leading order (NNLO) quantities use $\Lambda_{3,4,5,6}^{\overline{\mathrm{MS}}}=299.4,246,167.7,67.8 \mathrm{MeV}$, which yields $\alpha_{s}\left(5, M_{Z}^{2}\right)=0.114$. The structure functions are defined in Eqs.(3.9)-(3.17) of [6], where now $n_{f}=4$ and $m_{c}$ is replaced by $m_{b}=4.5 \mathrm{GeV} / \mathrm{c}^{2}$. To make this paper reasonably self-contained we now reproduce the final formulae we use for the structure functions. For $i=2, L$ the CSN scheme uses

$$
\begin{aligned}
& F_{i, Q}^{\mathrm{CSN}}\left(n_{f}+1, \Delta, Q^{2}, m^{2}\right)=e_{Q}^{2}\left[f_{Q+\bar{Q}}^{\mathrm{NNLO}}\left(n_{f}+1, \mu^{2}\right) \mathcal{C}_{i, Q}^{\mathrm{CSN}, \mathrm{NS},(0)}\left(\frac{Q^{2}}{m^{2}}\right)\right. \\
& +a_{s}\left(n_{f}+1, \mu^{2}\right)\left\{f_{Q+\bar{Q}}^{\mathrm{NLO}}\left(n_{f}+1, \mu^{2}\right) \otimes \mathcal{C}_{i, Q}^{\mathrm{CSN}, \mathrm{NS},(1)}\left(\frac{Q^{2}}{m^{2}}, \frac{Q^{2}}{\mu^{2}}\right)\right. \\
& \left.+f_{g}^{\mathrm{S}, \mathrm{NLO}}\left(n_{f}+1, \mu^{2}\right) \otimes \mathcal{C}_{i, g}^{\mathrm{CSN}, \mathrm{S},(1)}\left(\frac{Q^{2}}{m^{2}}, \frac{Q^{2}}{\mu^{2}}\right)\right\} \\
& +a_{s}^{2}\left(n_{f}+1, \mu^{2}\right)\left\{f _ { Q + \overline { Q } } ^ { \mathrm { LO } } ( n _ { f } + 1 , \mu ^ { 2 } ) \otimes \left(\mathcal{C}_{i, q}^{\mathrm{NS},(2)}\left(n_{f}+1, \frac{Q^{2}}{m^{2}}, \frac{Q^{2}}{\mu^{2}}\right)\right.\right. \\
& \left.+\mathcal{C}_{i, q}^{\mathrm{PS},(2)}\left(\frac{Q^{2}}{m^{2}}, \frac{Q^{2}}{\mu^{2}}\right)\right)+\sum_{l=1}^{n_{f}} f_{l+\bar{l}}^{\mathrm{LO}}\left(n_{f}+1, \mu^{2}\right) \otimes \mathcal{C}_{i, q}^{\mathrm{CSN}, \mathrm{PS},(2)}\left(\frac{Q^{2}}{m^{2}}, \frac{Q^{2}}{\mu^{2}}\right) \\
& \left.\left.+f_{g}^{\mathrm{S}, \mathrm{LO}}\left(n_{f}+1, \mu^{2}\right) \otimes \mathcal{C}_{i, g}^{\mathrm{CSN}, \mathrm{S},(2)}\left(\frac{Q^{2}}{m^{2}}, \frac{Q^{2}}{\mu^{2}}\right)\right\}\right] \\
& +a_{s}^{2}\left(n_{f}+1, \mu^{2}\right) \sum_{k=1}^{n_{f}} e_{k}^{2} f_{k+\bar{k}}^{\mathrm{LO}}\left(n_{f}+1, \mu^{2}\right) \otimes L_{i, q}^{\mathrm{HARD}, \mathrm{NS},(2)}\left(\Delta, \frac{Q^{2}}{m^{2}}\right) .
\end{aligned}
$$

where we choose the heavy quark $Q$ to be the bottom quark, so that the number of light flavors is $n_{f}=4$. The charge of the bottom quark is $e_{Q}=-1 / 3$ and its mass is $m=m_{b}$. The coefficient function $L_{i, q}^{\mathrm{HARD}}$ depends on a parameter $\Delta$ which refers to the invariant mass of the $Q \bar{Q}$-pair. For bottom quark production we choose $\Delta=100(\mathrm{GeV} / c)^{2}$. The coefficient functions labelled by $C^{\mathrm{CSN}}$ depend on the heavy quark mass but are finite in the limit $m \rightarrow 0$. They are defined in Eqs.(2.8)-(2.20) in [6]. To simplify the notation we 
will refer to the above structure functions as $F_{i, b}^{\mathrm{CSN}}\left(n_{f}=5\right)$, indicating that they depend on five-flavor parton densities.

The same parameters $e_{Q}, m_{b}, \Delta$ etc., also show up in the expressions for the bottom quark structure functions in the BMSN scheme. Here we have the representations

$$
\begin{aligned}
& F_{i, Q}^{\mathrm{BMSN}}\left(n_{f}+1, \Delta, Q^{2}, m^{2}\right)=F_{i, Q}^{\mathrm{EXACT}}\left(n_{f}, \Delta, Q^{2}, m^{2}\right) \\
& -F_{i, Q}^{\mathrm{ASYMP}}\left(n_{f}, \Delta, Q^{2}, m^{2}\right)+F_{i, Q}^{\mathrm{PDF}}\left(n_{f}+1, \Delta, Q^{2}, m^{2}\right) .
\end{aligned}
$$

The pieces in this formulae represent first the results in FOPT, given by

$$
\begin{aligned}
& F_{i, Q}^{\mathrm{EXACT}}\left(n_{f}, \Delta, Q^{2}, m^{2}\right)=e_{Q}^{2}\left[a_{s}\left(n_{f}, \mu^{2}\right) f_{g}^{\mathrm{S}, \mathrm{NLO}}\left(n_{f}, \mu^{2}\right) \otimes H_{i, g}^{\mathrm{S},(1)}\left(\frac{Q^{2}}{m^{2}}\right)\right. \\
& +a_{s}^{2}\left(n_{f}, \mu^{2}\right)\left\{\sum_{k=1}^{n_{f}} f_{k+\bar{k}}^{\mathrm{LO}}\left(n_{f}, \mu^{2}\right) \otimes H_{i, q}^{\mathrm{PS},(2)}\left(\frac{Q^{2}}{m^{2}}, \frac{Q^{2}}{\mu^{2}}\right)\right. \\
& \left.\left.+f_{g}^{\mathrm{S}, \mathrm{LO}}\left(n_{f}, \mu^{2}\right) \otimes H_{i, g}^{\mathrm{S},(2)}\left(\frac{Q^{2}}{m^{2}}, \frac{Q^{2}}{\mu^{2}}\right)\right\}\right] \\
& +a_{s}^{2}\left(n_{f}, \mu^{2}\right) \sum_{k=1}^{n_{f}} e_{k}^{2} f_{k+\bar{k}}^{\mathrm{LO}}\left(n_{f}, \mu^{2}\right) \otimes L_{i, q}^{\mathrm{HARD}, \mathrm{NS},(2)}\left(\Delta, \frac{Q^{2}}{m^{2}}\right) .
\end{aligned}
$$

The next pieces are the structure functions $F_{i, Q}^{\mathrm{ASYMP}}\left(n_{f}\right)$ which can be obtained from $F_{i, Q}^{\mathrm{EXACT}}\left(n_{f}\right)$ by replacing all exact heavy quark coefficient functions $H_{i, k}$ and $L_{i, k}(k=q, g)$ by their asymptotic analogues which are defined by

$$
H_{i, k}^{\mathrm{ASYMP}}=\lim _{Q^{2} \gg m^{2}} H_{i, k} \quad, \quad L_{i, k}^{\mathrm{ASYMP}}=\lim _{Q^{2} \gg m^{2}} L_{i, k} .
$$

Finally the structure functions $F_{i, Q}^{\mathrm{PDF}}\left(n_{f}+1\right)$ which are very often called the ZM-VFNS representations are defined by

$$
\begin{aligned}
& F_{i, Q}^{\mathrm{PDF}}\left(n_{f}+1, \Delta, Q^{2}, m^{2}\right)=e_{Q}^{2}\left[f_{Q+\bar{Q}}^{\mathrm{NNO}}\left(n_{f}+1, \mu^{2}\right) \mathcal{C}_{i, q}^{\mathrm{NS},(0)}\right. \\
& +a_{s}\left(n_{f}+1, \mu^{2}\right)\left\{f_{Q+\bar{Q}}^{\mathrm{NLO}}\left(n_{f}+1, \mu^{2}\right) \otimes \mathcal{C}_{i, q}^{\mathrm{NS},(1)}\left(\frac{Q^{2}}{\mu^{2}}\right)\right. \\
& \left.+f_{g}^{\mathrm{S}, \mathrm{NLO}}\left(n_{f}+1, \mu^{2}\right) \otimes \tilde{\mathcal{C}}_{i, g}^{\mathrm{S},(1)}\left(\frac{Q^{2}}{\mu^{2}}\right)\right\} \\
& +a_{s}^{2}\left(n_{f}+1, \mu^{2}\right)\left\{f _ { Q + \overline { Q } ^ { \mathrm { LO } } } ^ { \mathrm { LO } } ( n _ { f } + 1 , \mu ^ { 2 } ) \otimes \left(\mathcal{C}_{i, q}^{\mathrm{NS},(2)}\left(n_{f}+1, \frac{Q^{2}}{\mu^{2}}\right)\right.\right. \\
& \left.\left.+\tilde{\mathcal{C}}_{i, q}^{\mathrm{PS},(2)}\left(\frac{Q^{2}}{\mu^{2}}\right)\right)+\sum_{l=1}^{n_{f}} f_{l+\bar{l}}^{\mathrm{LO}}\left(n_{f}+1, \mu^{2}\right)\right) \otimes \tilde{\mathcal{C}}_{i, q}^{\mathrm{PS},(2)}\left(\frac{Q^{2}}{\mu^{2}}\right)
\end{aligned}
$$




$$
\begin{aligned}
& \left.\left.+f_{g}^{\mathrm{S}, \mathrm{LO}}\left(n_{f}+1, \mu^{2}\right) \otimes \tilde{\mathcal{C}}_{i, g}^{\mathrm{S},(2)}\left(\frac{Q^{2}}{\mu^{2}}\right)\right\}\right] \\
& +a_{s}^{2}\left(n_{f}+1, \mu^{2}\right) \sum_{k=1}^{n_{f}} e_{k}^{2} f_{k+\bar{k}}^{\mathrm{LO}}\left(n_{f}, \mu^{2}\right) \otimes L_{i, q}^{\mathrm{HARD}, \mathrm{ASYMP}, \mathrm{NS},(2)}\left(\Delta, \frac{Q^{2}}{m^{2}}\right)
\end{aligned}
$$

In all these results the heavy quark $Q$ refers to the bottom quark and the other parameters are defined above. For simplicity we will refer to these structure functions as $F_{i, b}^{\mathrm{EXACT}}\left(n_{f}=4\right)$, $F_{i, b}^{\mathrm{ASYMP}}\left(n_{f}=4\right)$, and $F_{i, b}^{\mathrm{PDF}}\left(n_{f}=5\right)$ respectively, which indicates that the first two structure functions depend on four-flavor densities and the last one depends on five-flavor densities. The parton densities $f_{k}$ in the above formulae are represented in leading order (LO), nextto-leading order (NLO) and next-to-next-to-leading order (NNLO). The NNLO case refers to the boundary conditions imposed in [6] since the three-loop splitting functions are not known yet. These parton densities have been constructed in [8] starting from a three-flavor parametrization in [7]. The multiplication of the densities with the heavy and light parton coefficient functions is done in such a way that the perturbation series is strictly truncated at order $\alpha_{s}^{2}$. This is necessary to avoid scheme dependent terms which would otherwise arise beyond order $\alpha_{s}^{2}$. Therefore the following requirement is satisfied

$$
F_{i, Q}^{\mathrm{CSN}}\left(n_{f}=5\right)=F_{i, Q}^{\mathrm{BMSN}}\left(n_{f}=5\right)=F_{i, Q}^{\mathrm{EXACT}}\left(n_{f}=4\right) \quad \text { for } \quad Q^{2} \leq m^{2} .
$$

Since $f_{Q}\left(m^{2}\right)^{\mathrm{NNLO}} \neq 0$ (see [2]) this condition can be only satisfied when we truncate the perturbation series at the same order. Furthermore because of Eq. (II.4) and the property

$$
\lim _{Q^{2} \gg m^{2}} \mathcal{C}_{i, k}^{\mathrm{CSN},(1)}\left(n_{f}+1, \frac{Q^{2}}{m^{2}}, \frac{Q^{2}}{\mu^{2}}\right)=\mathcal{C}_{i, k}^{(l)}\left(n_{f}+1, \frac{Q^{2}}{\mu^{2}}\right),
$$

we have the asymptotic relation

$$
\begin{aligned}
& \lim _{Q^{2} \gg m^{2}} F_{i, Q}^{\mathrm{BMSN}}\left(n_{f}+1, \Delta, Q^{2}, m^{2}\right)=\lim _{Q^{2} \gg m^{2}} F_{i, Q}^{\mathrm{CSN}}\left(n_{f}+1, \Delta, Q^{2}, m^{2}\right) \\
& =F_{i, Q}^{\mathrm{PDF}}\left(n_{f}+1, \Delta, Q^{2}, m^{2}\right) .
\end{aligned}
$$

At first sight the form of the expression for $F_{i, Q}^{\mathrm{BMSN}}$ in Eq. (II.2) looks quite different from the one presented for $F_{i, Q}^{\mathrm{CSN}}$ in Eq. ([1.1). However this is not true. Using the mass factorization relations for the asymptotic heavy quark coefficient functions in [2] one can cast $F_{i, Q}^{\mathrm{BMSN}}$ into the same form as presented for $F_{i, Q}^{\mathrm{CSN}}$ where all quark coefficient functions of the type $\mathcal{C}_{i, Q}^{\mathrm{CSN}}$ are replaced by their light quark analogues $\mathcal{C}_{i, q}$ appearing in Eq. (III.5). This replacement also applies to the $\mathcal{C}_{i, Q}^{\mathrm{CSN}}$ occurring in the mass factorization relations for $\mathcal{C}_{i, g}^{\mathrm{CSN}, \mathrm{S}}$ and $\mathcal{C}_{i, q}^{\mathrm{CSN}, \mathrm{PS}}$ presented in [6]. Therefore the difference between the CSN and BMSN schemes can be attributed to the powers $\left(m^{2} / Q^{2}\right)^{j}$ showing up in $\mathcal{C}_{i, Q}^{\mathrm{CSN}}$ but absent in $\mathcal{C}_{i, q}$. This effect is only noticeable in the threshold region where $Q^{2} \sim m^{2}$ as we will show below.

The heavy quark coefficient functions $\mathcal{C}_{i, k}^{\mathrm{CSN}}, H_{i, k}, L_{i, k}(k=Q, q, g)$ and the light partonic coefficient functions $\mathcal{C}_{i, k}(k=q, g)$ can be traced back to the following processes 


$$
\begin{aligned}
& \mathcal{C}_{i, g}^{\mathrm{CSN}, \mathrm{S},(1)}, H_{i, g}^{\mathrm{S},(1)}: \gamma^{*}+g \rightarrow Q+\bar{Q} \quad \text { [6] (CSN), [1] (EXACT), } \\
& \text { [11] (ASYMP) } \\
& \mathcal{C}_{i, g}^{\mathrm{CSN}, \mathrm{S},(2)}, H_{i, g}^{\mathrm{S},(2)}: \gamma^{*}+g \rightarrow Q+\bar{Q}+g \text { [6] (CSN), [1] (EXACT), } \\
& \text { [1] (ASYMP) } \\
& \mathcal{C}_{i, q}^{\mathrm{CSN}, \mathrm{PS},(2)}, H_{i, q}^{\mathrm{PS},(2)}: \gamma^{*}+q \rightarrow Q+\bar{Q}+q \quad \text { Bethe-Heitler reaction } \\
& \text { [6] (CSN), [1] (EXACT), [11] (ASYMP) } \\
& L_{i, q}^{\mathrm{HARD}, \mathrm{NS},(2)}: \gamma^{*}+q \rightarrow Q+\bar{Q}+q \quad \text { Compton reaction } \\
& \text { [6] (EXACT and ASYMP) } \\
& \mathcal{C}_{i, Q}^{\mathrm{CSN}, \mathrm{NS},(0)}, H_{i, Q}^{\mathrm{NS},(0)}: \gamma^{*}+Q \rightarrow Q \\
& \mathcal{C}_{i, Q}^{\mathrm{CSN}, \mathrm{NS},(1)}, H_{i, Q}^{\mathrm{NS},(1)}: \gamma^{*}+Q \rightarrow Q+g \\
& \mathcal{C}_{i, q}^{\mathrm{NS},(0)}: \gamma^{*}+q \rightarrow q \\
& \mathcal{C}_{i, q}^{\mathrm{NS},(1)}: \gamma^{*}+q \rightarrow q+g \\
& \mathcal{C}_{i, q}^{\mathrm{NS},(2)}: \gamma^{*}+q \rightarrow q+g+g \quad 13 \\
& \mathcal{C}_{i, q}^{\mathrm{NS},(2)}: \gamma^{*}+q \rightarrow q+\bar{q}+q \\
& \tilde{\mathcal{C}}_{i, q}^{\mathrm{PS},(2)}: \gamma^{*}+q \rightarrow q+\bar{q}+q \\
& \tilde{\mathcal{C}}_{i, g}^{\mathrm{S},(1)}: \gamma^{*}+g \rightarrow q+\bar{q} \\
& \tilde{\mathcal{C}}_{i, g}^{\mathrm{S},(2)}: \gamma^{*}+g \rightarrow q+\bar{q}+g \quad \text { [13]. }
\end{aligned}
$$

Behind the reactions we have quoted the references in which the corresponding coefficient functions can be found. Note that the heavy quark coefficient functions $H_{i, k}$ are mass singular when $m \rightarrow 0$. This can be observed immediately when one looks at $H_{i, k}^{\mathrm{ASYMP}}$ which behaves like $\ln ^{m}\left(\mu^{2} / m^{2}\right) \ln ^{n}\left(Q^{2} / m^{2}\right)$ (see [11]). After the logarithms are removed one obtains the quantities $\mathcal{C}_{i, k}^{\text {CNS }}$ which, even though they depend on $m$, are finite in the limit $m \rightarrow 0$. The coefficient function $L_{i, k}^{\mathrm{HARD}}$ is finite by itself because as we mentioned above we have imposed a lower cut off $\Delta=100(\mathrm{GeV} / c)^{2}$ on the invariant mass of the $Q \bar{Q}$-pair. Finally notice that all parton densities, coefficient functions and the running coupling constant are presented in the $\overline{\mathrm{MS}}$-scheme.

Now we present results for the various structure functions. We are interested in the bottom quark structure functions $F_{i, b}^{\mathrm{CSN}}\left(n_{f}=5\right)$ and $F_{i, b}^{\mathrm{BMSN}}\left(n_{f}=5\right)$ for $i=2, L$ in NNLO for the CSN [6] and BMSN [2] schemes respectively. In Fig. 1 we have plotted the structure 
functions $F_{2, b}^{\mathrm{CSN}}\left(n_{f}=5\right), F_{2, b}^{\mathrm{BMSN}}\left(n_{f}=5\right), F_{2, b}^{\mathrm{PDF}}\left(n_{f}=5\right)$ and $F_{2, b}^{\mathrm{EXACT}}\left(n_{f}=4\right)$ in the region $20<Q^{2}<10^{3}$ in units of $(\mathrm{GeV} / \mathrm{c})^{2}$ for $x=0.05$. This figure reveals that there is hardly any difference between the BMSN and CSN prescriptions. The curves in both prescriptions are essentially identical to that for $F_{2, b}^{\mathrm{EXACT}}\left(n_{f}=4\right)$. In this region $F_{2, b}^{\mathrm{PDF}}\left(n_{f}=5\right)$ is larger than the other results which is expected from the discussion of the bottom quark density given in [8]. There is still an appreciable difference at the highest plotted $Q^{2}$ demonstrating that mass effects are important up to very large scales. Notice that for $Q^{2} \leq 35(\mathrm{GeV} / \mathrm{c})^{2}$ $F_{2, b}^{\mathrm{PDF}}\left(n_{f}=5\right)$ becomes negative which means that bottom quark electroproduction cannot be described by this quantity anymore. In Fig. 2 we present the same plots for $x=0.005$. Again one cannot distinguish between $F_{2, b}^{\mathrm{BMSN}}\left(n_{f}=5\right)$ and $F_{2, c}^{\mathrm{CSN}}\left(n_{f}=5\right)$ but now both are smaller than $F_{2, b}^{\mathrm{EXACT}}\left(n_{f}=4\right)$ over the whole $Q^{2}$ range. The latter is smaller than $F_{2, b}^{\mathrm{PDF}}\left(n_{f}=5\right)$ in particular for $Q^{2}>35(\mathrm{GeV} / \mathrm{c})^{2}$. Further we want to emphasize that due to our careful treatment of the threshold region there is an excellent cancellation between $F_{2, b}^{\mathrm{PDF}}\left(n_{f}=5\right)$ and $F_{2, b}^{\mathrm{ASYMP}}\left(n_{f}=4\right)$ so that both $F_{2, b}^{\mathrm{CSN}}\left(n_{f}=5\right)$ and $F_{2, b}^{\mathrm{BMSN}}\left(n_{f}=5\right)$ tend to $F_{2, b}^{\mathrm{EXACT}}\left(n_{f}=4\right)$ at $Q^{2}=m_{b}^{2}$. At large $Q^{2}$ we have a cancellation between $F_{2, b}^{\mathrm{ASYMP}}\left(n_{f}=4\right)$ and $F_{2, b}^{\mathrm{EXACT}}\left(n_{f}=4\right)$ so that both $F_{2, b}^{\mathrm{CSN}}\left(n_{f}=5\right)$ and $F_{2, b}^{\mathrm{BMSN}}\left(n_{f}=5\right)$ slowly tend to $F_{2, b}^{\mathrm{PDF}}\left(n_{f}=5\right)$. They are only identical at extremely large $Q^{2}$ demonstrating that mass effects are still important over a wide range in $x$ and $Q^{2}$.

In Fig. 3 we show similar plots as in Fig.1 for the bottom quark longitudinal structure functions. Here we observe a small difference between the plots for $F_{L, b}^{\mathrm{CSN}}\left(n_{f}=5\right)$ and $F_{L, b}^{\mathrm{BMSN}}\left(n_{f}=5\right)$ in the region $20<Q^{2}<10^{3}(\mathrm{GeV} / \mathrm{c})^{2}$. Furthermore $F_{L, b}^{\mathrm{PDF}}\left(n_{f}=5\right)$ is considerably larger than the other three structure functions, which differs from the behavior seen in Fig.1. This can be mainly attributed to the gluon density which plays a more prominant role in $F_{L, b}$ than in $F_{2, b}$. For $x=0.005$ (see Fig.4) the small difference between the BMSN and the CSN descriptions becomes more conspicuous for low $Q^{2}$.

In Figs.5 and 6 we make a comparison between the NLO and the NNLO structure functions $F_{2, b}^{\mathrm{CSN}}\left(n_{f}=5\right)$ and $F_{2, b}^{\mathrm{BMSN}}\left(n_{f}=5\right)$. Both the CSN and and BMSN descriptions lead to the same results in both NLO and NNLO. However while going from NLO to NNLO the the structure functions $F_{2, b}^{\mathrm{CSN}}\left(n_{f}=5\right)$ and $F_{2, b}^{\mathrm{BMSN}}\left(n_{f}=5\right)$ increase a little bit. The differences in the case of $x=0.005$ in Fig. 6 are even smaller than those observed for $x=0.05$ in Fig.5. The same comparison between NLO and NNLO results is made for the longitudinal structure functions in Figs.7 and 8. Here the differences between NLO and NNLO cases are much larger than in the case of $F_{2, b}$ in Figs.5,6. In NLO both $F_{L, b}^{\mathrm{BMSN}}\left(n_{f}=5\right)$ and $F_{L, b}^{\mathrm{CSN}}\left(n_{f}=5\right)$ are smaller than the NNLO results.

Previous results for $F_{i, b}^{\mathrm{EXACT}}\left(x, Q^{2}, m_{b}^{2}\right)$ and have been presented in Figs. 20a,20b in [1] for a now obsolete set of parton densities, so the values quoted there are too small. To show these changes we add in Figs. 9 and 10 plots for the $x$ dependence of $F_{2, b}^{\mathrm{CSN}}\left(n_{f}=5\right)$, $F_{2, b}^{\mathrm{BMSN}}\left(n_{f}=5\right), F_{2, b}^{\mathrm{PDF}}\left(n_{f}=5\right)$ and $F_{2, b}^{\mathrm{EXACT}}\left(n_{f}=4\right)$ at fixed $Q^{2}=30$ and $Q^{2}=100$ in units of $(\mathrm{GeV} / \mathrm{c})^{2}$ respectively. Finally we also show in Figs. 11 and 12 plots for the $x$ dependence of $F_{L, b}^{\mathrm{CSN}}\left(n_{f}=5\right), F_{L, b}^{\mathrm{BMSN}}\left(n_{f}=5\right), F_{L, b}^{\mathrm{PDF}}\left(n_{f}=5\right)$ and $F_{L, b}^{\mathrm{EXACT}}\left(n_{f}=4\right)$ at fixed $Q^{2}=30$ and $Q^{2}=100$ in units of $(\mathrm{GeV} / \mathrm{c})^{2}$ respectively. Note that there are also some recent results for $F_{2, b}$ in [5] in the TR scheme and in [14] for FOPT.

The plots for $F_{L, b}^{\mathrm{CSN}}\left(n_{f}=5\right)$ in Figs.7,8 do not show a negative region at small $Q^{2}$ which could have been expected by analogy with the results for $F_{L, c}^{\mathrm{CSN}}\left(n_{f}=4\right)$ in [6]. In the case of bottom quark production the negative regions do occur but at even smaller values of $x$. 
In Figs. 13, 14 we show the same plots as in Figs.1,3 respectively but for $x=5 \times 10^{-5}$. Now the structure function $F_{L, b}^{\mathrm{CSN}}$ is negative in the region $Q^{2} \approx 30(\mathrm{GeV} / \mathrm{c})^{2}$. In Figs. 15,16 we show the same plots as in Figs. 5,7 respectively but for $x=5 \times 10^{-5}$. Fig. 16 shows that the longitudinal structure functions for the case of bottom production also have negative regions at small $Q^{2}$ values in both NLO and NNLO. This phenomenon also occurs for the charm quark structure functions in [6]. In the NLO case this arises because the term $f_{g}^{\mathrm{S}, \mathrm{NLO}}\left(n_{f}+1, \mu^{2}\right) \otimes \mathcal{C}_{L, g}^{\mathrm{CSN}, \mathrm{S},(1)}\left(Q^{2} / m^{2}, Q^{2} / \mu^{2}\right)$ in Eq. (III.1) is negative due to the definition of the gluon coefficient function in the CSN scheme (see Eq.(2.19)) in [6]) which is given by

$$
\begin{aligned}
\mathcal{C}_{L, g}^{\mathrm{CSN}, \mathrm{S},(1)}\left(\frac{Q^{2}}{m^{2}}, \frac{Q^{2}}{\mu^{2}}\right) & =H_{L, g}^{\mathrm{S},(1)}\left(\frac{Q^{2}}{m^{2}}\right)-A_{Q g}^{\mathrm{S},(1)}\left(\frac{\mu^{2}}{m^{2}}\right) \mathcal{C}_{L, Q}^{\mathrm{CSN}, \mathrm{NS},(0)}\left(\frac{Q^{2}}{m^{2}}\right), \\
\text { with } \quad \mathcal{C}_{L, Q}^{\mathrm{CSN}, \mathrm{NS},(0)} & =\frac{4 m^{2}}{Q^{2}},
\end{aligned}
$$

where $A_{Q g}^{\mathrm{S},(1)}$ denotes the one-loop operator matrix element computed in [11]. Notice that the latter and the lowest order exact coefficient function $H_{L, g}^{\mathrm{S},(1)}$ are always positive. Because of the minus sign in Eq. (ㅍ.10) it appears that the coefficient function $\mathcal{C}_{L, g}^{\mathrm{CSN}, \mathrm{S},(1)}$ can become negative in particular at low $Q^{2}$ values. In the NNLO case one obtains more negative contributions due to the term $f_{Q+\bar{Q}}^{\mathrm{NNLO}}\left(n_{f}+1, \mu^{2}\right) \mathcal{C}_{L, Q}^{\mathrm{CSN}, \mathrm{NS},(0)}$ in formula (II.1). It turns out that $f_{Q+\bar{Q}}^{\mathrm{NNLO}}\left(n_{f}+1, x, \mu^{2}\right)$ is negative at small $x$ and $\mu^{2}=Q^{2} \geq m^{2}$. Notice that at the latter scale $f_{Q+\bar{Q}}^{\mathrm{LO}}\left(n_{f}+1, x, \mu^{2}\right)$ and $f_{Q+\bar{Q}}^{\mathrm{NLO}}\left(n_{f}+1, x, \mu^{2}\right)$ are very small because they vanish at $\mu=m$ in contrast to $f_{Q+Q}^{\mathrm{NNLO}}\left(n_{f}+1, x, \mu^{2}\right)$. The behavior of the structure function above is characteristic of the CSN scheme since it does not appear in the case of BMSN. This is because in the latter scheme the longitudinal coefficient function, represented by $\mathcal{C}_{L, q}^{\mathrm{CSN}, \mathrm{NS},(0)}$, is identical to zero so that the zeroth order contribution to $F_{L, b}^{\mathrm{BMSN}}\left(n_{f}=5\right)$ vanishes and the first order correction is given by $\mathcal{C}_{L, g}^{\mathrm{BMSN}, \mathrm{S},(1)}=H_{L, g}^{\mathrm{S},(1)}$. The latter leads to a positive structure function over the whole kinematical region. To further demonstrate this point we plot in Fig. 17 pieces of the NLO result

$$
\begin{aligned}
& F_{L, b}^{\mathrm{CSN}}\left(n_{f}+1, Q^{2}, m^{2}\right)=e_{b}^{2}\left[f_{b+\bar{b}}^{\mathrm{NLO}}\left(n_{f}+1, \mu^{2}\right) \mathcal{C}_{L, b}^{\mathrm{CSN}, \mathrm{NS},(0)}\left(\frac{Q^{2}}{m^{2}}\right)\right. \\
& +a_{s}\left(n_{f}+1, \mu^{2}\right)\left\{f_{b+\bar{b}}^{\mathrm{LO}}\left(n_{f}+1, \mu^{2}\right) \otimes \mathcal{C}_{L, b}^{\mathrm{CSN}, \mathrm{NS},(1)}\left(\frac{Q^{2}}{m^{2}}, \frac{Q^{2}}{\mu^{2}}\right)\right. \\
& \left.+f_{g}^{\mathrm{S}, \mathrm{LO}}\left(n_{f}+1, \mu^{2}\right) \otimes \mathcal{C}_{L, g}^{\mathrm{CSN}, \mathrm{S},(1)}\left(\frac{Q^{2}}{m^{2}}, \frac{Q^{2}}{\mu^{2}}\right)\right\} .
\end{aligned}
$$

The sum of the b-quark contributions, labelled Term1, is always positive. The gluonic contribution, labelled Term2, is clearly negative over a wide range in $Q^{2}$ and large enough that the sum $F_{L, b}^{\mathrm{CSN}}$ is also negative for $30 \leq Q^{2} \leq 150 \mathrm{GeV}^{2}$, as in Fig.16. This behavior is due to the gluonic coefficient function $\mathcal{C}_{L, g}^{\mathrm{CSN}, \mathrm{S},(1)}$ which is explained below Eq. (II.10). However the magnitude of the gluonic contribution depends on the choice of the gluon density. If we use 
an NLO gluon density in the order $\alpha_{s}$ contribution to the structure function in Eq. ([I.11) rather than a LO gluon density then the sum of the first two terms is unchanged but the gluonic part is now smaller in magnitude. These contributions are shown in Fig.18 where now $\mid$ Term2 $\mid \leq$ Term1 so that the total result for $F_{L, b}^{C S N}$ is everywhere positive. However this procedure violates our prescription for the computation of the structure functions in both the CSN and the BMSN schemes. In this prescription the LO densities are multiplied by the highest order coefficient function whereas the NLO densities are combined with lower order coefficient functions (see formulae ([1.1), ([I.3) and ([1.5)). In this way the perturbation series is truncated up to the order we want to compute the structure functions. Hence we avoid terms, arising beyond that order, which introduce a scheme dependence and spoils the threshold behavior (see [6]). The latter happens if one follows the usual procedure where one multiplies the highest order densities by the highest order coefficient functions. The difference between the usual procedure and our prescription is not only shown by our parton density set but is also observed for other sets presented in the literature. Examples are recent sets such as MRST98 [9] (with $m_{b}=4.3 \mathrm{GeV}, m_{c}=1.35 \mathrm{GeV}$ ), MRST99 [15] (with $m_{b}=4.3 \mathrm{GeV}, m_{c}=1.43 \mathrm{GeV}$ ), and CTEQ5 [10] (with $m_{b}=4.5 \mathrm{GeV}, m_{c}=1.3 \mathrm{GeV}$ ). Note that the MRST99 set does not provide LO densities. Using their NLO densities they yield positive values for the $Q^{2}$ dependence of $F_{L, b}^{\mathrm{CSN}}$ at $x=5 \times 10^{-5}$. There are both LO (CTEQ5L) and NLO (CTEQ5M) densities in the CTEQ5 set and we have checked that, for the same $x, Q^{2}$ values $F_{L, b}^{\mathrm{CSN}}$ is positive with purely NLO densities but has a negative region when the LO and NLO densities are used according to our prescription. The observations made above leads to the conclusion that the $4 \mathrm{~m}^{2} / Q^{2}$ term in the non-singlet CSN longitudinal coefficient function in Eq. ([1.10) leads to a negative gluonic coefficient function. When the latter is used together with the latest LO and NLO parton density sets $F_{L, b}^{\mathrm{CSN}}\left(n_{f}=5\right)$ becomes negative in the low $Q^{2}$-region at small $x$. We speculate that the CSN scheme would always yield positive structure functions if we could use parton density sets which fitted data with convolutions of LO densities with $O\left(\alpha_{s}\right)$ coefficient functions and NLO densities with zeroth order coefficient functions. Unfortunately such densities are not available.

To summarize the main points we have implemented two variable flavor number schemes for bottom quark electroproduction in NNLO and compared them with NLO FOPT results. The schemes differ in the way mass factorization is implemented. In the CSN scheme this is done with respect to the full heavy and light quark structure functions at finite $Q^{2}$. In the BMSN scheme the mass factorization is only applied to the coefficient functions in the large $Q^{2}$ limit. Both schemes require four-flavor and five-flavor parton densities which satisfy discontinuous NNLO matching conditions at a scale $\mu=m_{b}$. We have constructed these densities using our own evolution code [8]. The schemes also require matching conditions on the coefficient functions which are implemented in this paper. Note that we have also removed the dangerous terms in $\ln ^{3}\left(Q^{2} / m_{b}^{2}\right)$ from the Compton contributions so that both $F_{i, b}^{\mathrm{CSN}}\left(n_{f}=5\right)$ and $F_{i, b}^{\mathrm{BMSN}}\left(n_{f}=5\right)$ are collinear safe. As in [6] we have done this in a way which is consistent with our study of inclusive quantities by implementing a cut $\Delta$ on the mass of the $b-\bar{b}$ pair. We stress that any ZM-VFNS bottom quark density description of $F_{i, b}$ must use collinear safe definitions. This is not required in the fixed order perturbation theory approach given by $F_{i, b}^{\mathrm{EXACT}}\left(n_{f}=4\right)$ in $[1]$ for moderate $Q^{2}$-values.

Finally we made a careful analysis of the threshold behaviors of $F_{i, b}^{\mathrm{CSN}}\left(n_{f}=5\right)$ and 
$F_{i, b}^{\mathrm{BMSN}}\left(n_{f}=5\right)$. In order to achieve the required cancellations at the scale $\mu=m_{b}$ so that they both become equal to $F_{i, b}^{\mathrm{EXACT}}\left(n_{f}=4\right)$ one must be very careful to combine terms with the same order in the expansion in $\alpha_{s}$. The approximation we made in this paper, of using NLO splitting functions in place of NNLO splitting functions, was sufficient for our purposes. We successfully implemented the required cancellations near threshold and the corresponding limits at large scales came out naturally. Inconsistent sets of parton densities automatically spoil these cancelations. Since there are only minor differences between the CSN, BMSN and NLO FOPT predictions it is clear that the use of variable flavor number schemes for bottom quark production is not required for the analysis of HERA data. However the ZM-VFNS description is clearly inadequate at small $Q^{2}$.

\section{ACKNOWLEDGMENTS}

The work of A. Chuvakin and J. Smith was partially supported by the National Science Foundation grant PHY-9722101. The work of W.L. van Neerven was supported by the EC network 'QCD and Particle Structure' under contract No. FMRX-CT98-0194. 


\section{REFERENCES}

[1] E. Laenen, S. Riemersma, J. Smith and W.L. van Neerven, Nucl. Phys. B392, 162 (1993); ibid. 229 (1993); S. Riemersma, J. Smith and W.L. van Neerven, Phys. Lett. B347, 43 (1995); B.W. Harris and J. Smith, Nucl. Phys. B452, 109 (1995).

[2] M. Buza, Y. Matiounine, J. Smith, W.L. van Neerven, Eur. Phys. J. C1, 301 (1998).

[3] M.A.G. Aivazis, J.C. Collins, F.I. Olness and W.-K. Tung, Phys. Rev. D50, 3102 (1994); F. Olness and S. Riemersma, Phys. Rev. D51, 4746 (1995).

[4] M. Buza, Y. Matiounine, J. Smith, W.L. van Neerven, Phys. Lett. B411, 211 (1997); W.L. van Neerven, Acta Phys. Polon. B28, 2715 (1997);

W.L. van Neerven in Proceedings of the 6th International Workshop on Deep Inelastic Scattering and QCD "DIS98" edited by GH. Coremans and R. Roosen, (World Scientific, 1998), p. 162-166, hep-ph/9804445;

J. Smith in New Trends in HERA Physics, edited by B.A. Kniehl, G. Kramer and A. Wagner, (World Scientific, 1998), p. 283, hep-ph/9708212.

[5] R.S. Thorne and R.G. Roberts, Phys. Lett. B421, 303 (1998); Phys. Rev. D57, 6871 (1998).

[6] A. Chuvakin, J. Smith and W.L. van Neerven, hep-ph/9910250.

[7] M. Glück, E. Reya and A. Vogt, Eur. Phys. J. C5, 461 (1998).

[8] A. Chuvakin and J. Smith, hep-ph/9911504.

[9] A.D. Martin, R.G. Roberts, W.J. Stirling and R. Thorne, Eur. Phys. J. C4, 463 (1998).

[10] H.L. Lai, J. Huston, S. Kuhlmann, J. Morfín, F. Olness, J. Owens, J. Pumplin, W.K. Tung, hep-ph/9903282.

[11] M. Buza, Y. Matiounine, J. Smith, R. Migneron and W.L. van Neerven, Nucl. Phys. B472, 611 (1996).

[12] W.L. van Neerven and J.A.M. Vermaseren, Nucl. Phys. B238, 73 (1984).

[13] W.L. van Neerven and E.B. Zijlstra, Phys. Lett. B272, 127 (1991); E.B. Zijlstra and W.L. van Neerven, Phys. Lett. B273, 476 (1991); Nucl. Phys. B383, 525 (1992).

[14] K. Daum, S. Riemersma, B.W. Harris, E. Laenen and J. Smith, in Proceedings of the Workshop 1995/1996 on Future Physics at HERA, Hamburg, 1996. Edited by G. Ingelman, A. De Roeck and R. Klanner, (Deutsches Electronen-Synchrotron, Hamburg 1996) p 89, hep-ph/9609478.

[15] A.D. Martin, R.G. Roberts, W.J. Stirling and R. Thorne, hep-ph/9907231. 


\section{Figure Captions}

Fig. 1. The bottom quark structure functions $F_{2, b}^{\mathrm{EXACT}}\left(n_{f}=4\right)$ (solid line) $F_{2, b}^{\mathrm{CSN}}\left(n_{f}=5\right)$, (dot-dashed line) $F_{2, b}^{\mathrm{BMSN}}\left(n_{f}=5\right)$, (dashed line) and $F_{2, b}^{\mathrm{PDF}}\left(n_{f}=5\right)$, (dotted line) in NNLO for $x=0.05$ plotted as functions of $Q^{2}$.

Fig. 2. Same as in Fig. 1 but now for $x=0.005$.

Fig. 3. The bottom quark structure functions $F_{L, b}^{\mathrm{EXACT}}\left(n_{f}=4\right)$ (solid line) $F_{L, b}^{\mathrm{CSN}}\left(n_{f}=5\right)$, (dot-dashed line) $F_{L, b}^{\mathrm{BMSN}}\left(n_{f}=5\right)$, (dashed line) and $F_{L, b}^{\mathrm{PDF}}\left(n_{f}=5\right)$, (dotted line) in NNLO for $x=0.05$ plotted as functions of $Q^{2}$.

Fig. 4. Same as in Fig. 3 but now for $x=0.005$.

Fig. 5. The bottom quark structure functions $F_{2, b}^{\mathrm{BMSN}}\left(n_{f}=5\right)$ in NLO (solid line), NNLO (dotted line) and $F_{2, b}^{\mathrm{CSN}}\left(n_{f}=5\right)$ in NLO (dashed line), NNLO (dot-dashed line) for $x=0.05$ plotted as functions of $Q^{2}$.

Fig. 6. Same as in Fig. 5 but now for $x=0.005$.

Fig. 7. The bottom quark structure functions $F_{L, b}^{\mathrm{BMSN}}\left(n_{f}=5\right)$ in NLO (solid line), NNLO (dotted line) and $F_{L, b}^{\mathrm{CSN}}\left(n_{f}=5\right)$ in NLO (dashed line), NNLO (dot-dashed line) for $x=0.05$ plotted as functions of $Q^{2}$.

Fig. 8. Same as in Fig. 7 but now for $x=0.005$.

Fig. 9. The bottom quark structure functions $F_{2, b}^{\mathrm{EXACT}}\left(n_{f}=4\right)$ (solid line) $F_{2, b}^{\mathrm{CSN}}\left(n_{f}=5\right)$, (dot-dashed line) $F_{2, b}^{\mathrm{BMSN}}\left(n_{f}=5\right)$, (dashed line) and $F_{2, b}^{\mathrm{PDF}}\left(n_{f}=5\right)$, (dotted line) in NNLO for $Q^{2}=30(\mathrm{GeV} / \mathrm{c})^{2}$ plotted as functions of $x$.

Fig. 10. Same as in Fig. 9 but now for $Q^{2}=100(\mathrm{GeV} / \mathrm{c})^{2}$.

Fig. 11. The bottom quark structure functions $F_{L, b}^{\mathrm{EXACT}}\left(n_{f}=4\right)$ (solid line) $F_{L, b}^{\mathrm{CSN}}\left(n_{f}=5\right)$, (dot-dashed line) $F_{L, b}^{\mathrm{BMSN}}\left(n_{f}=5\right)$, (dashed line) and $F_{L, b}^{\mathrm{PDF}}\left(n_{f}=5\right)$, (dotted line) in NNLO for $Q^{2}=30(\mathrm{GeV} / \mathrm{c})^{2}$ plotted as functions of $x$.

Fig. 12. Same as in Fig. 11 but now for $Q^{2}=100(\mathrm{GeV} / \mathrm{c})^{2}$.

Fig. 13. Same as in Fig. 1 but now for $x=5 \times 10^{-5}$.

Fig. 14. Same as in Fig. 3 but now for $x=5 \times 10^{-5}$.

Fig. 15. Same as in Fig. 5 but now for $x=5 \times 10^{-5}$.

Fig. 16. Same as in Fig. 7 but now for $x=5 \times 10^{-5}$.

Fig. 17. The bottom quark structure function $F_{L, b}^{\mathrm{EXACT}}\left(n_{f}=4\right)$ (solid line) $F_{L, b}^{\mathrm{CSN}}\left(n_{f}=5\right)$, (dot-dashed line) together with the NLO charm density piece Term1 (dotted line) and the LO gluon density piece Term2 (dashed line), see text, for $x=5 \times 10^{-5}$ plotted as functions of $Q^{2}$. 
Fig. 18. The bottom quark structure function $F_{L, b}^{\mathrm{EXACT}}\left(n_{f}=4\right)$ (solid line) $F_{L, b}^{\mathrm{CSN}}\left(n_{f}=5\right)$, (dot-dashed line) together with the NLO charm density piece Term1 (dotted line) and the NLO gluon density piece Term2 (dashed line), see text, for $x=5 \times 10^{-5}$ plotted as functions of $Q^{2}$. 
Fig 1

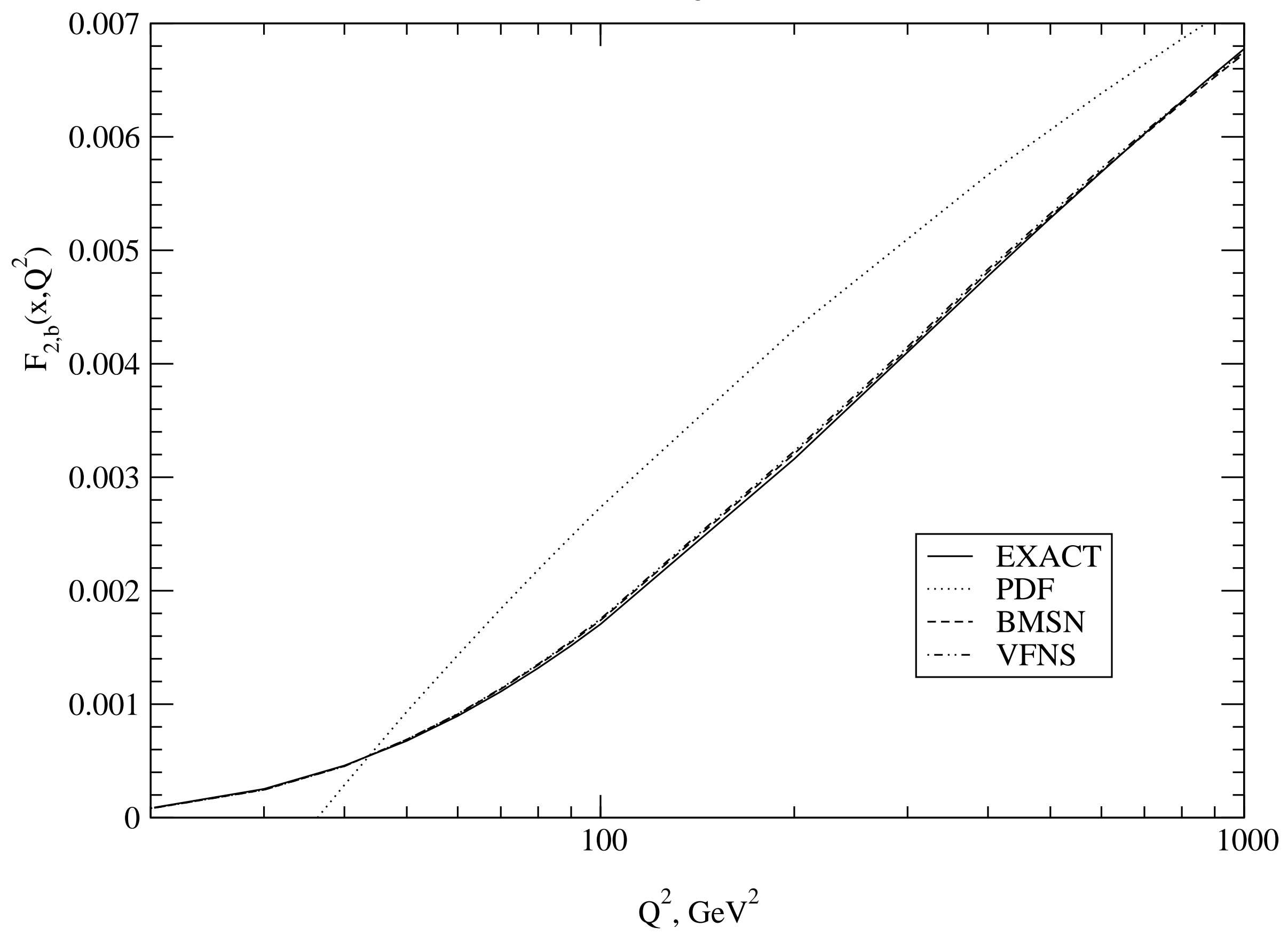


Fig 2

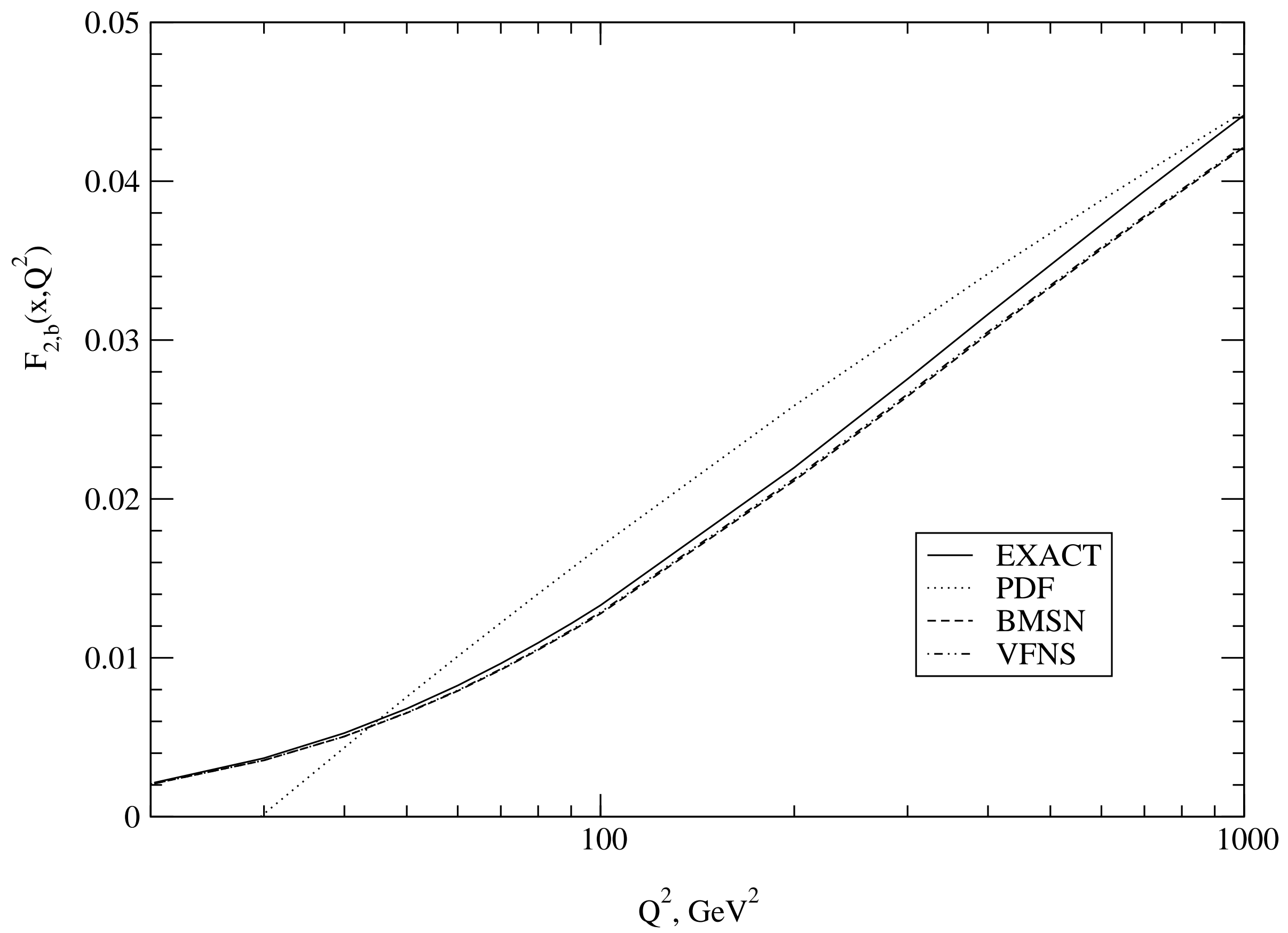


Fig 3

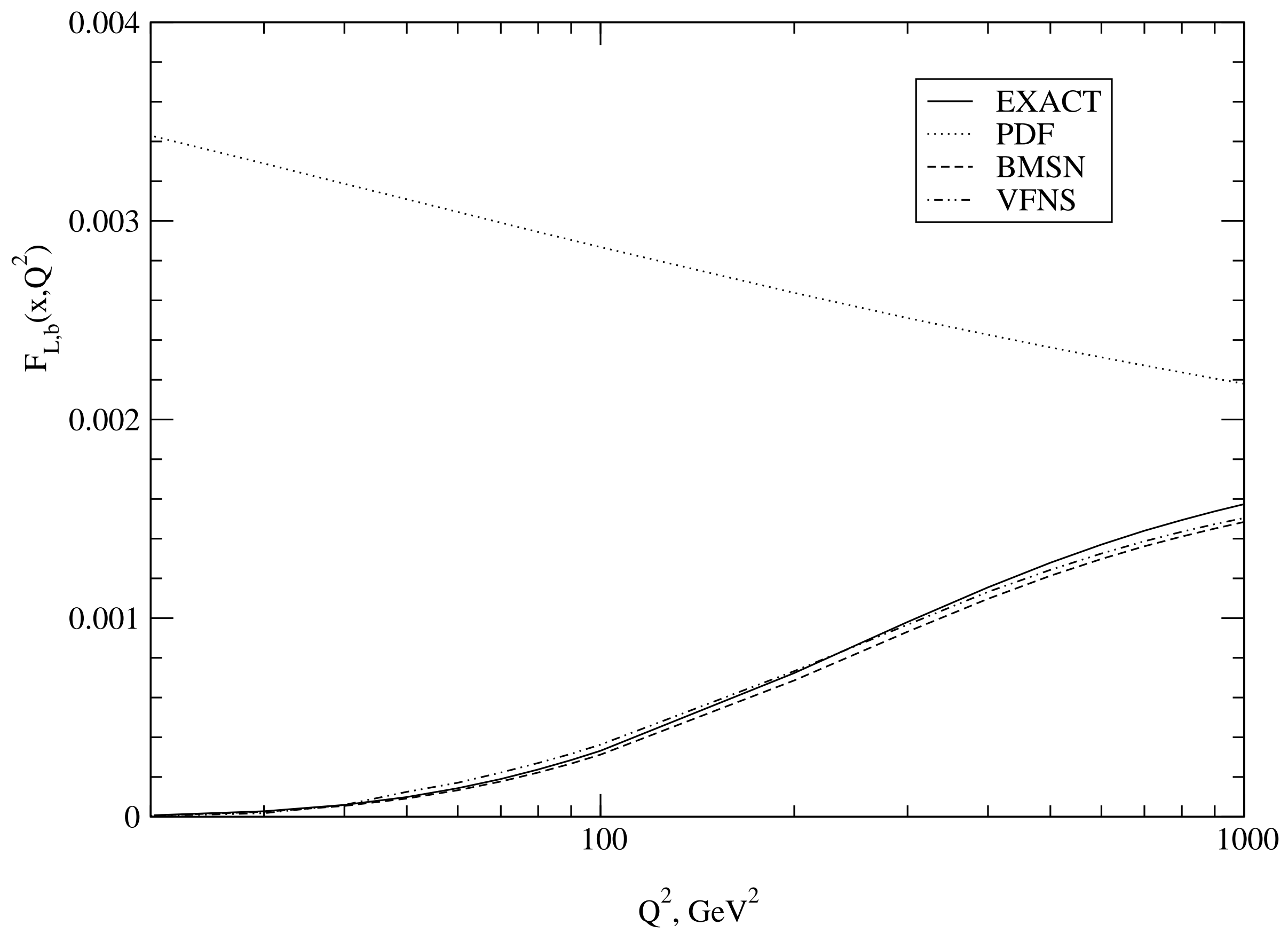


Fig 4

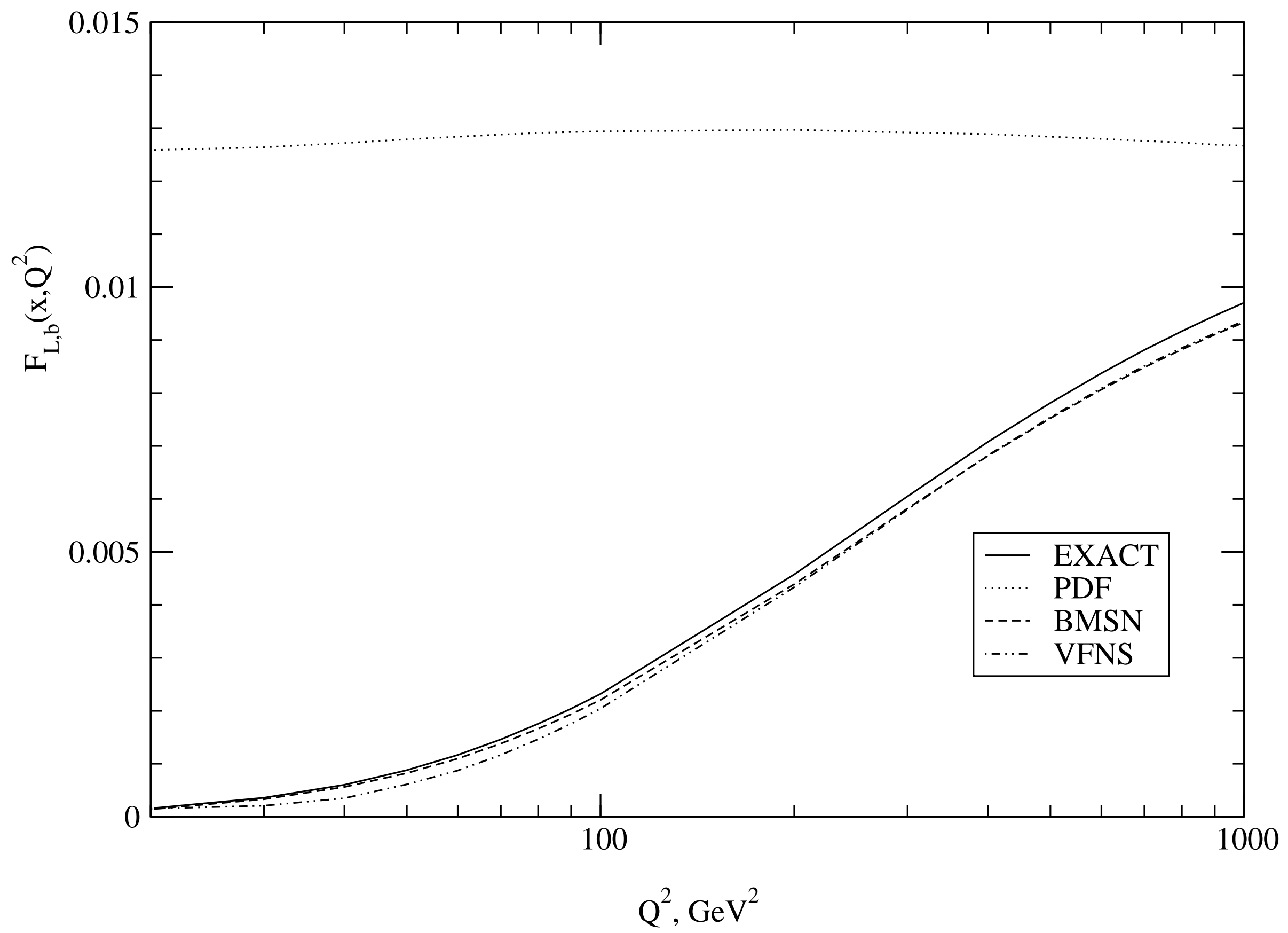


Fig 5

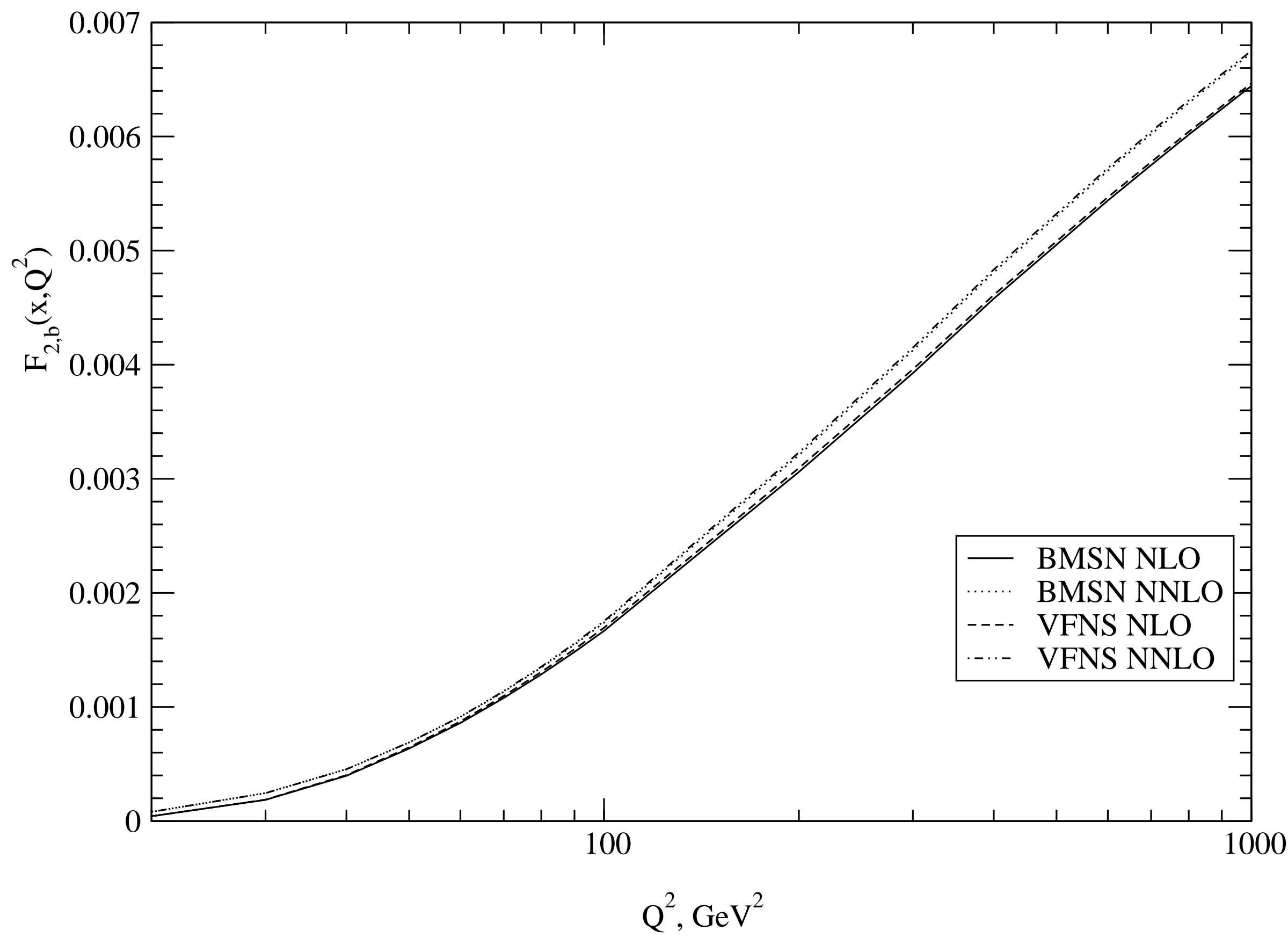


Fig 6

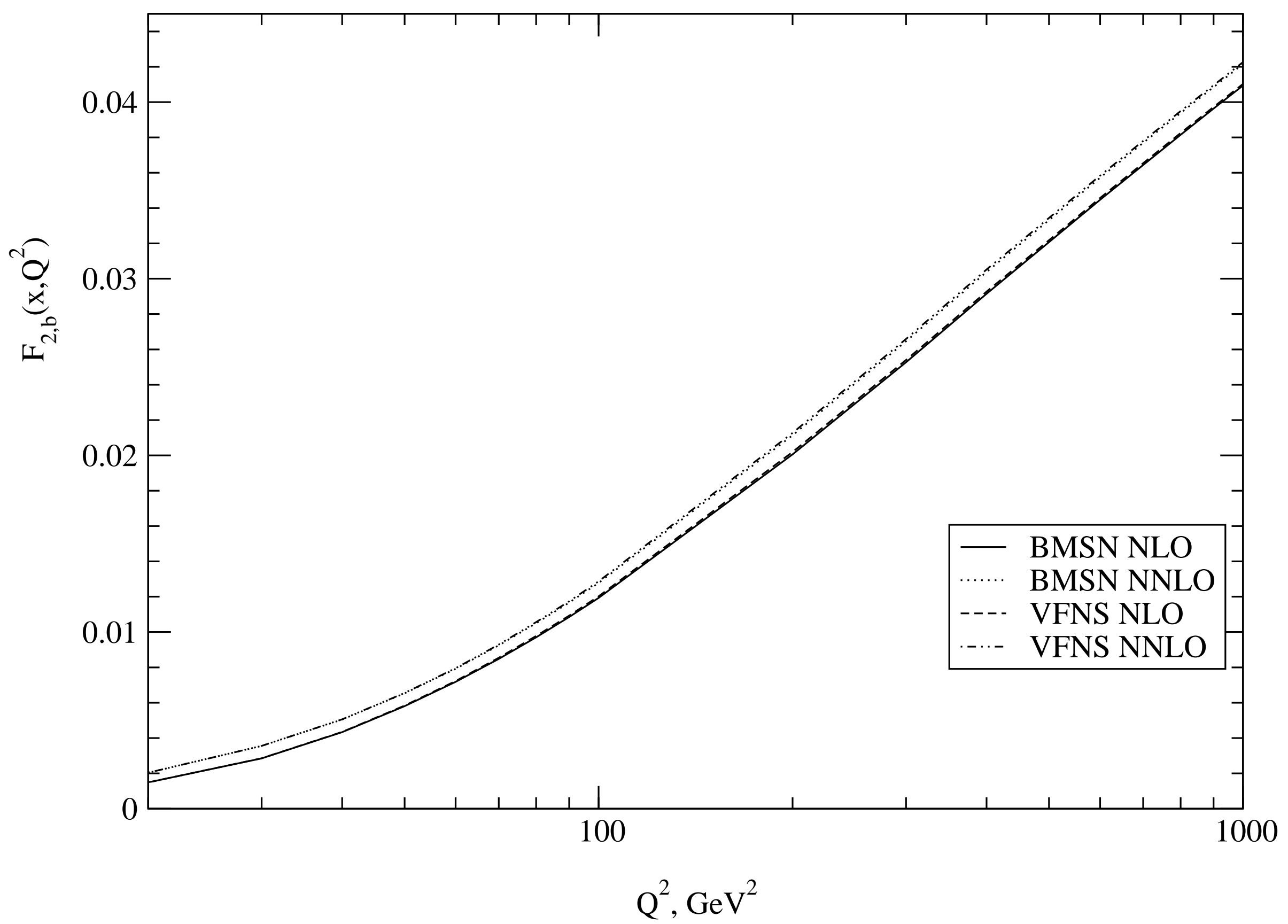


Fig 7

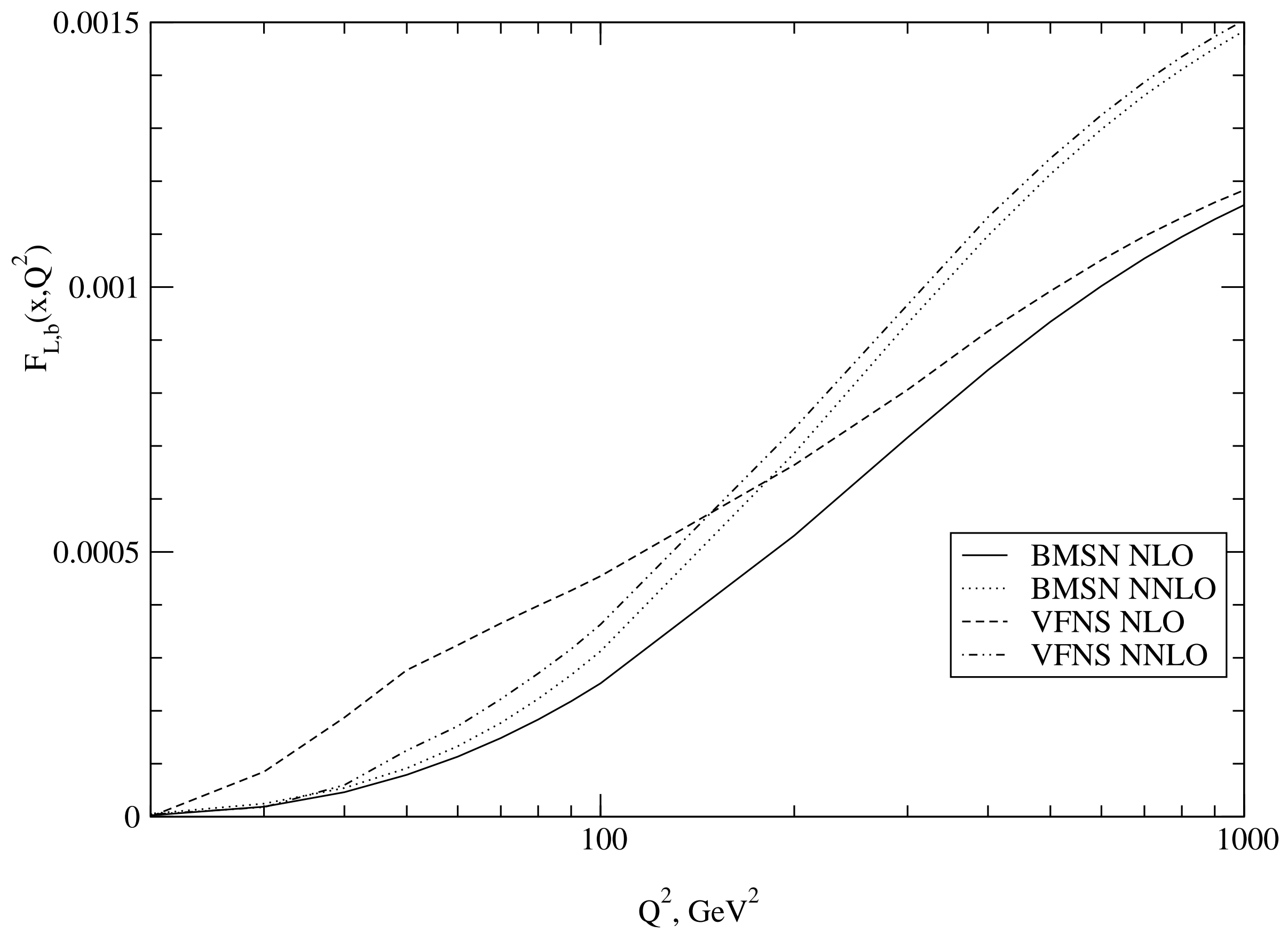


Fig 9

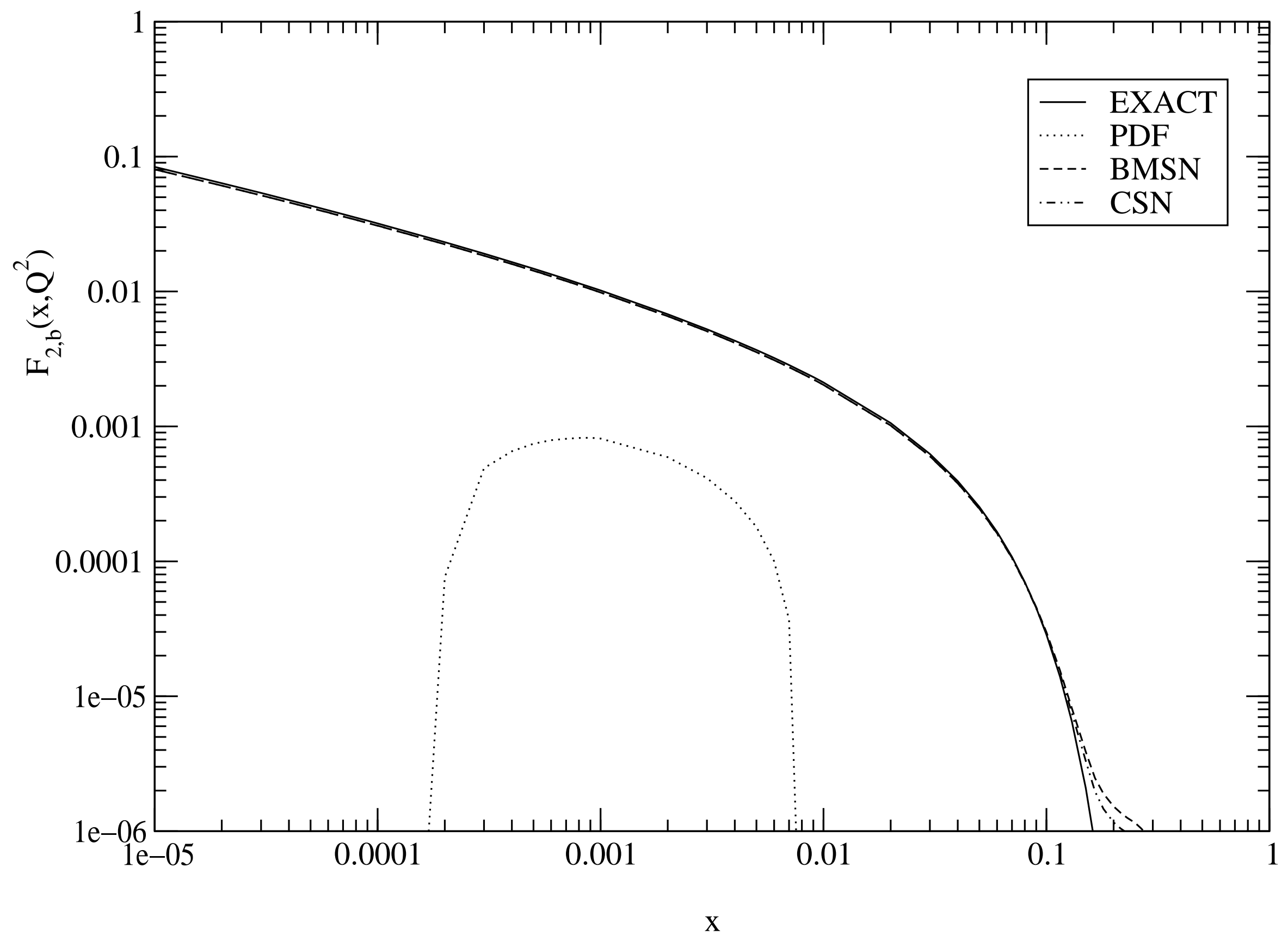


Fig 10

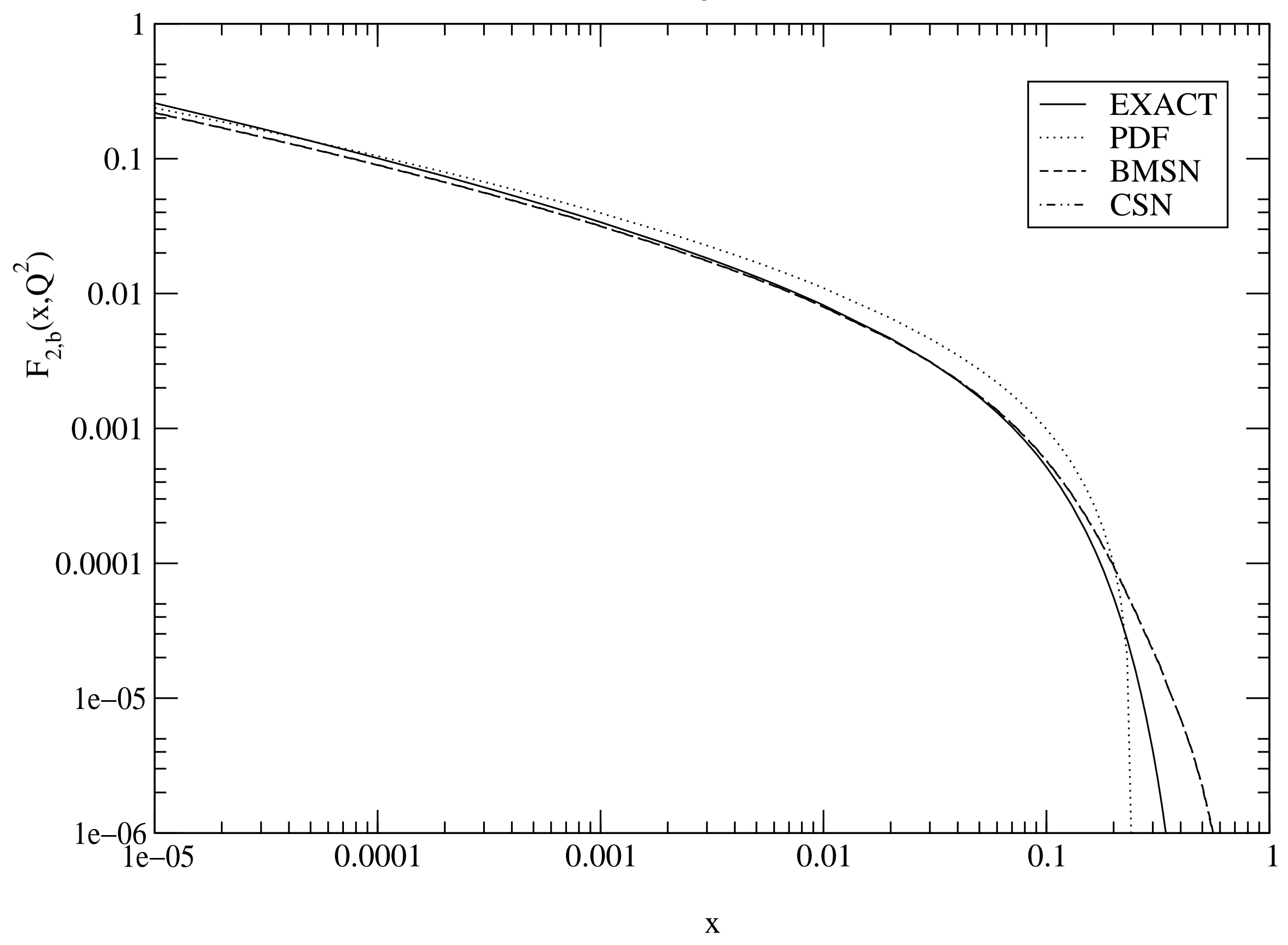


Fig 11

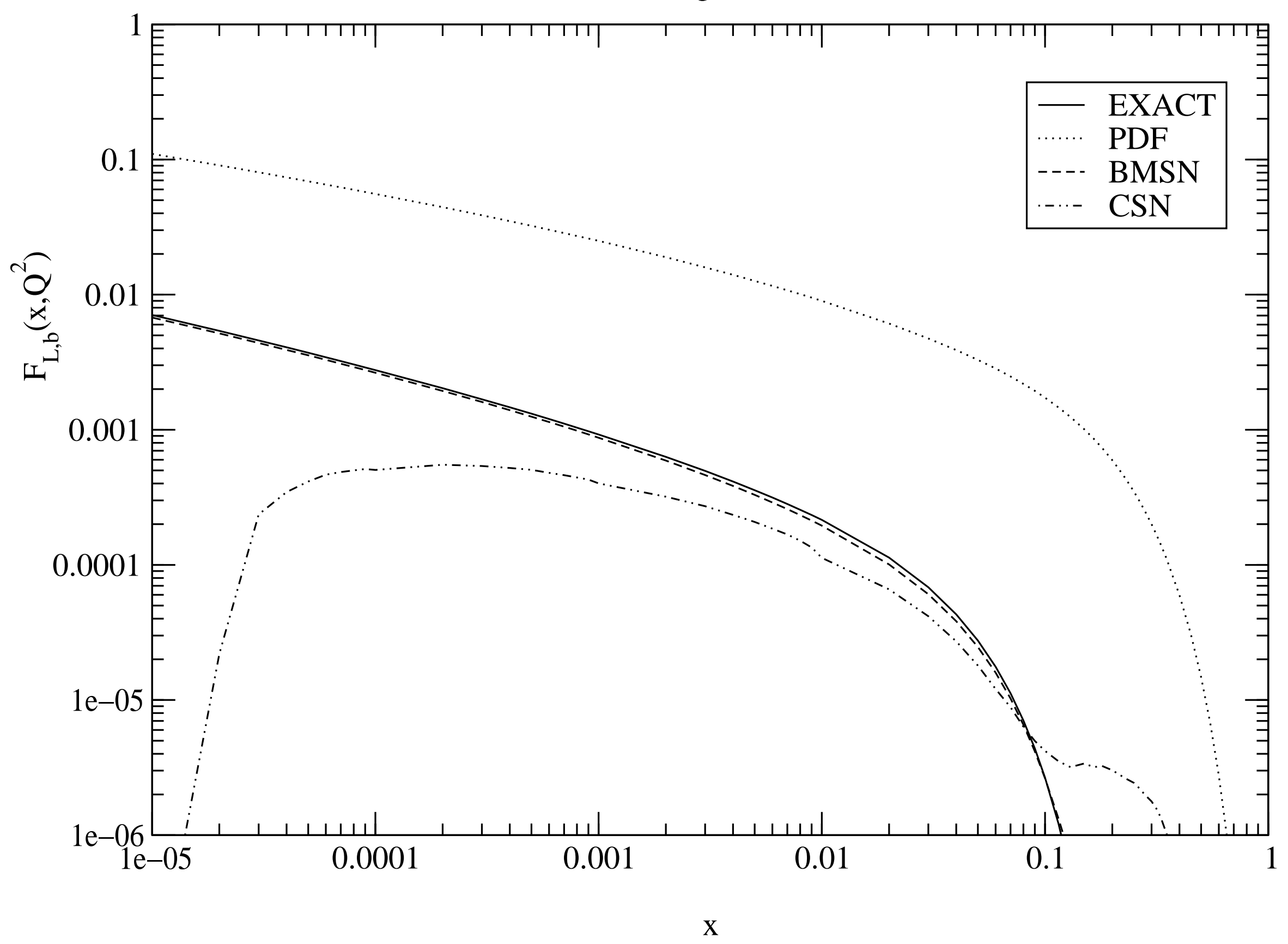


Fig 12

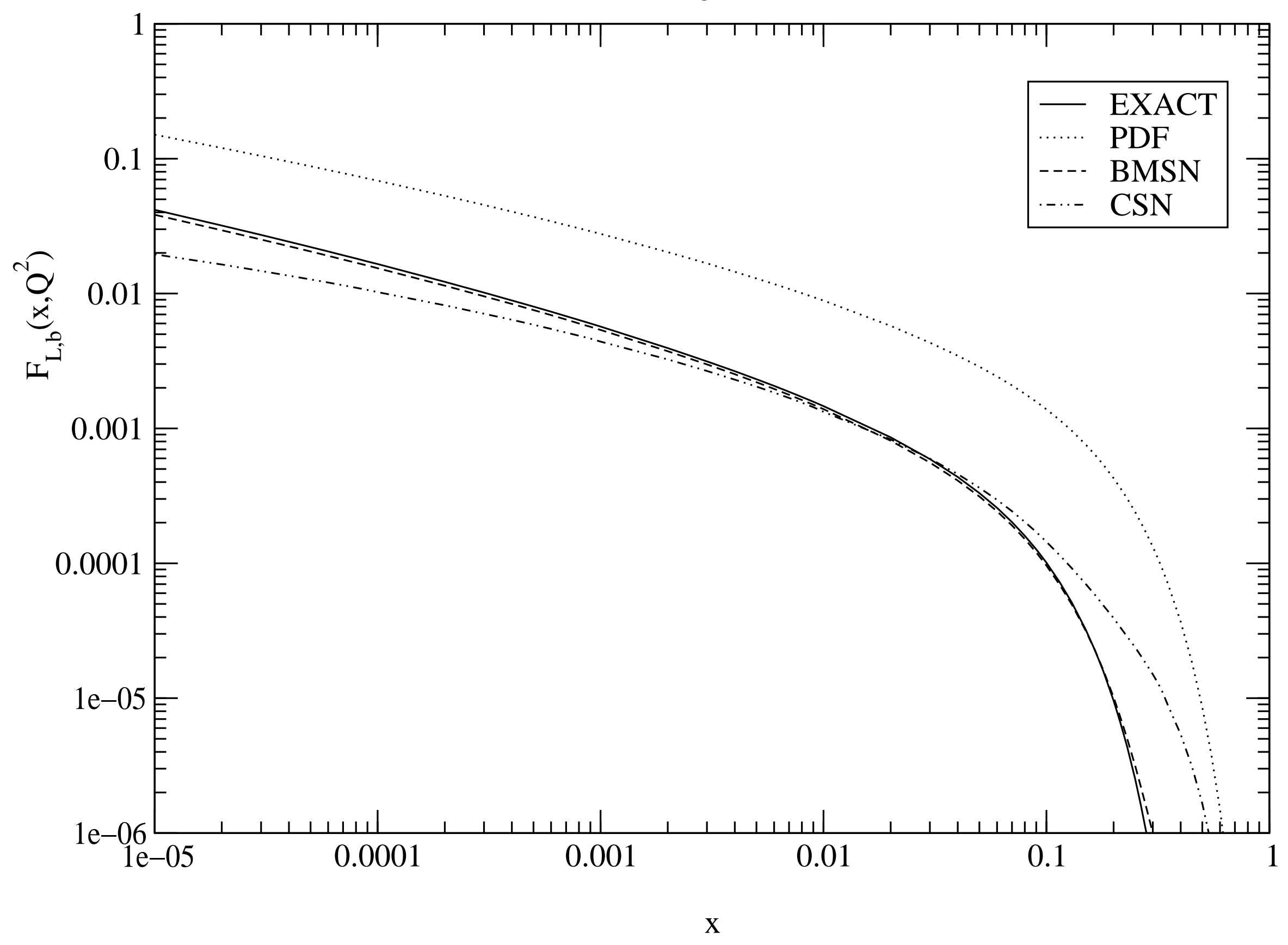


Fig 13

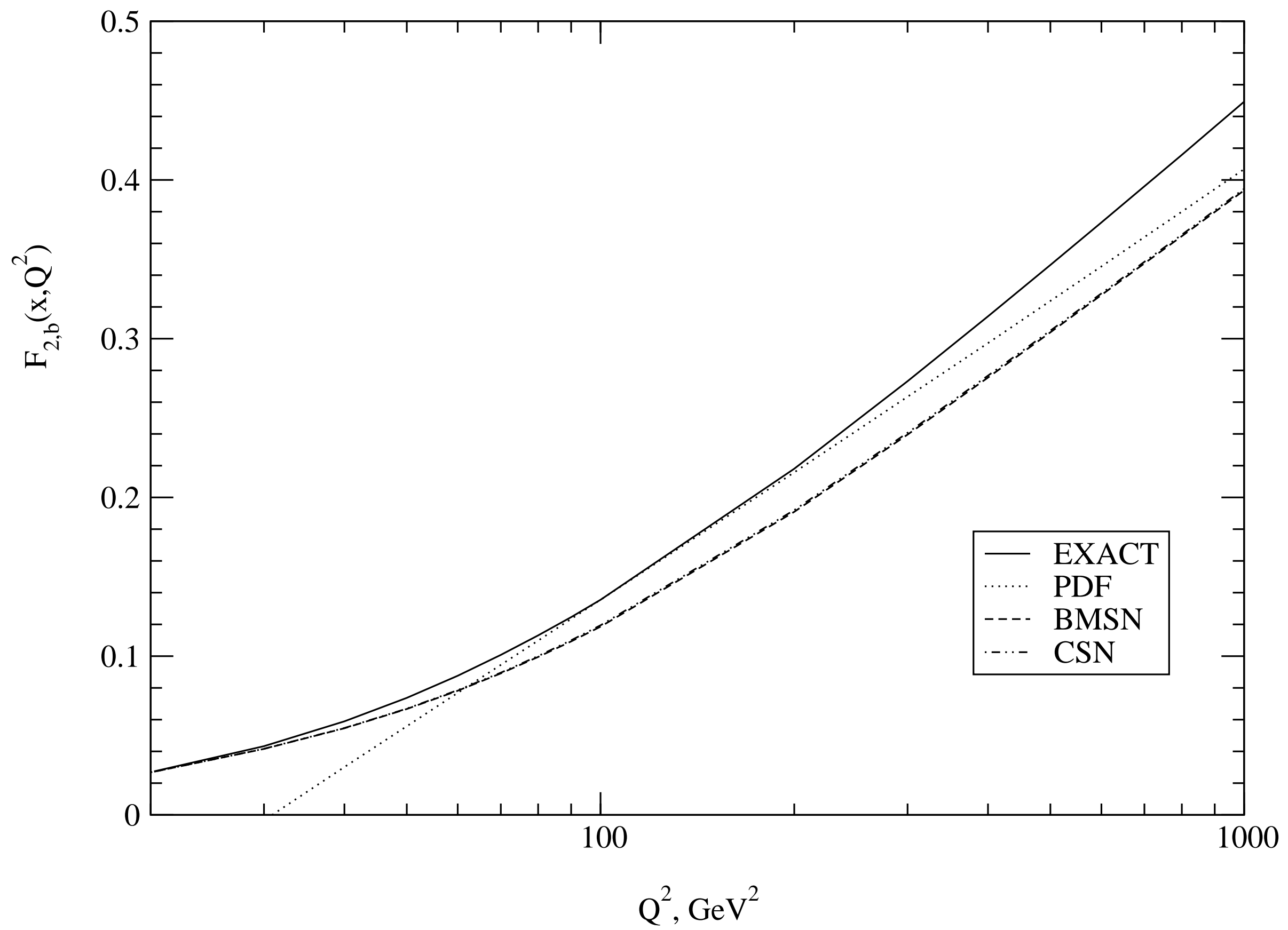


Fig 14

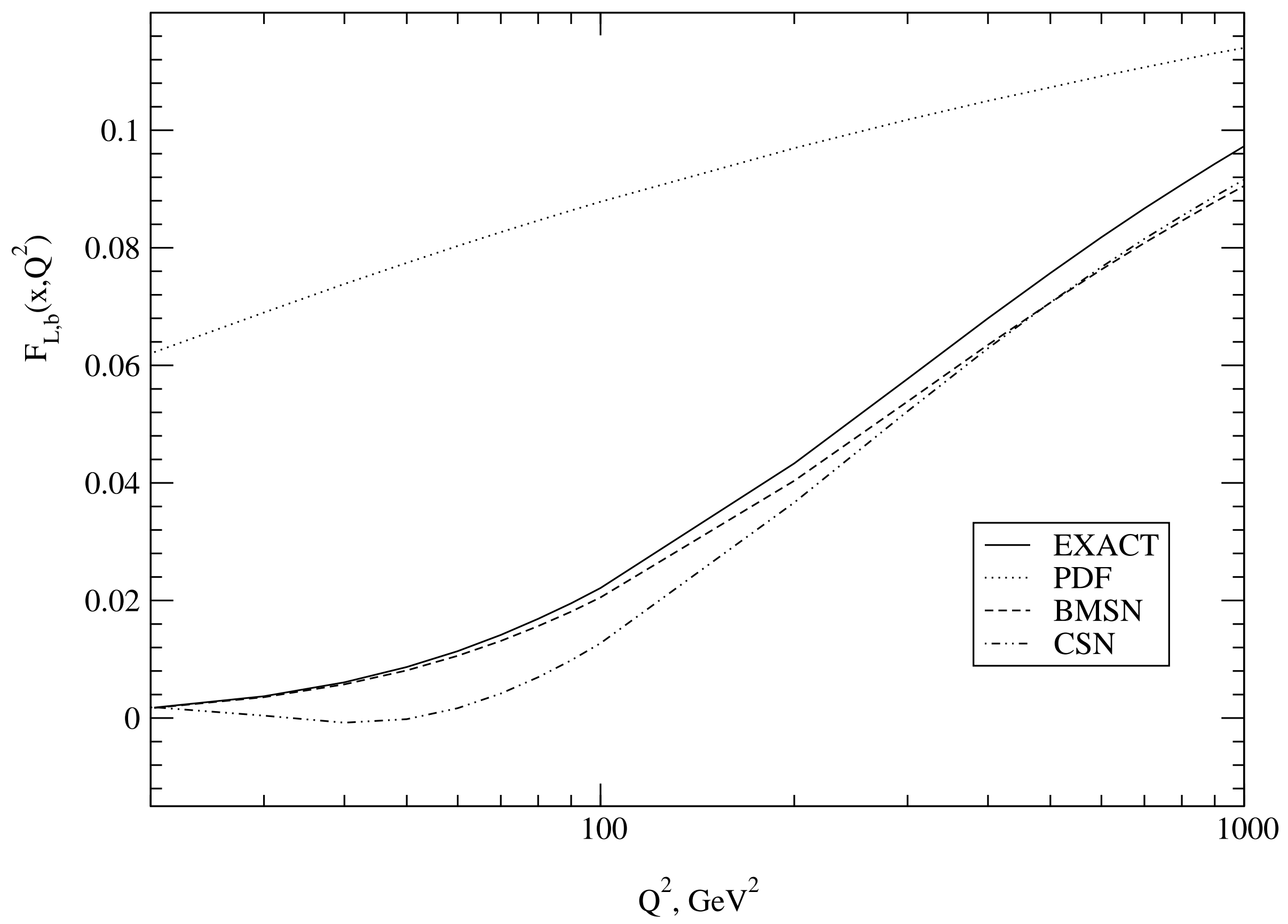


Fig 15

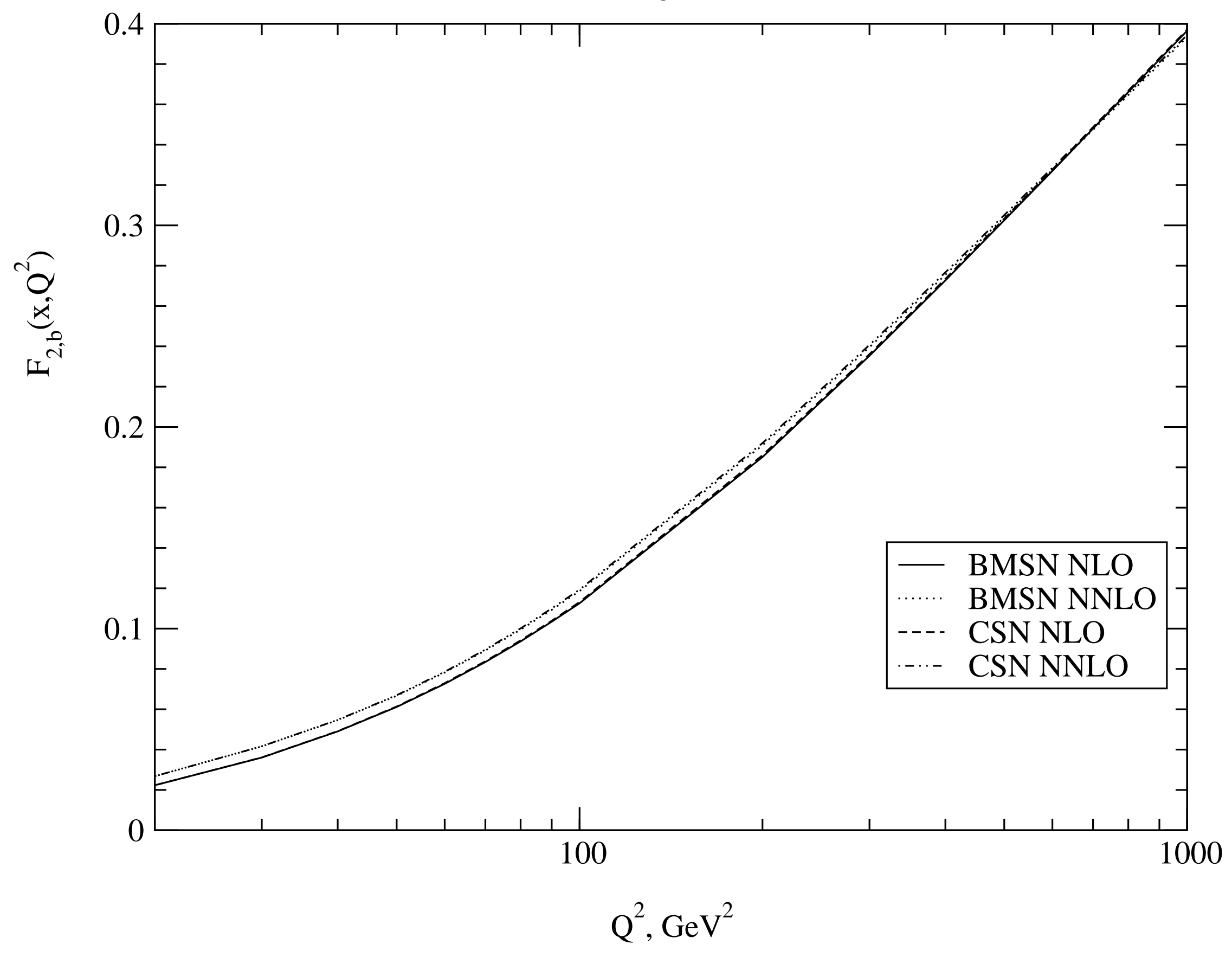


Fig 18

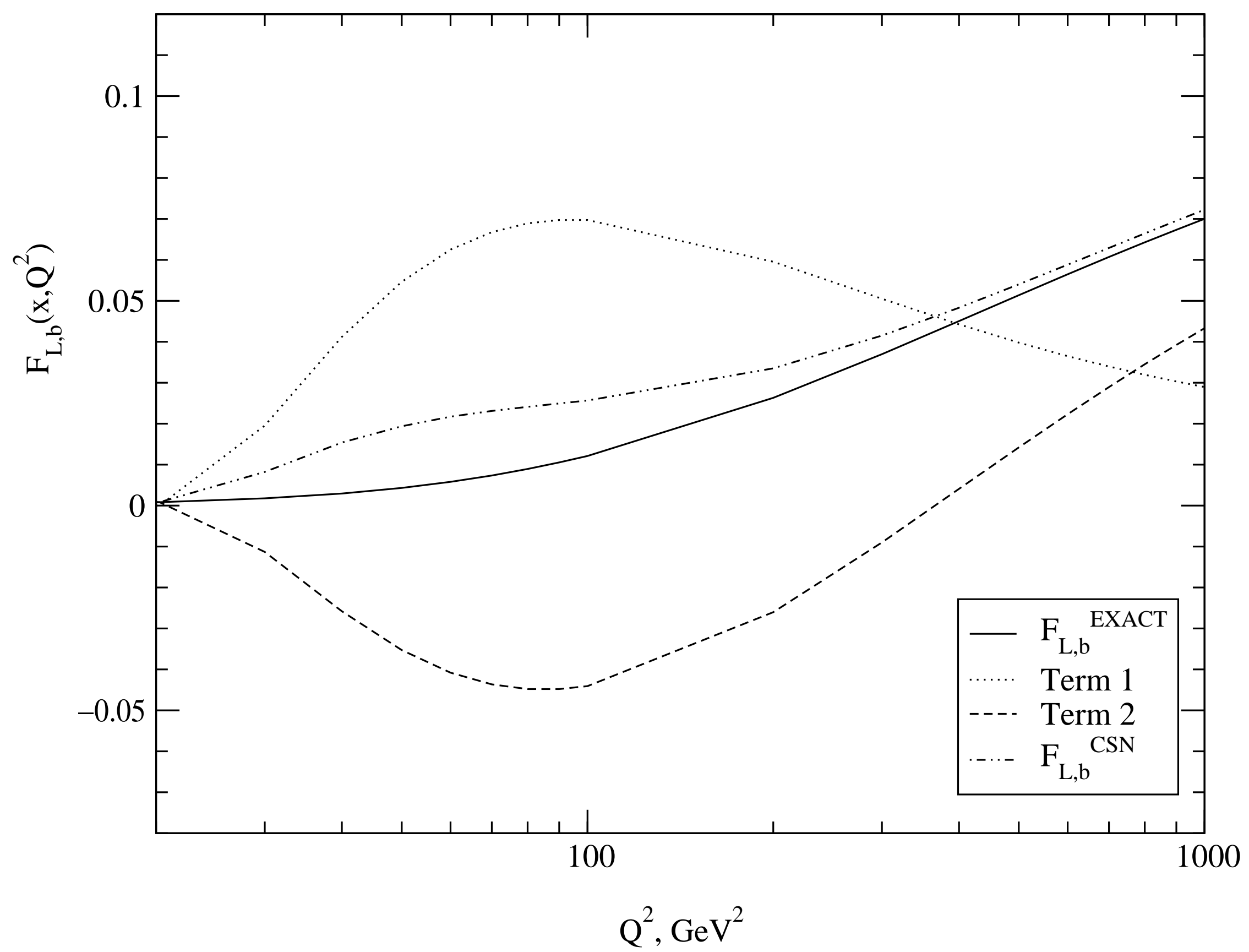

